\title{
LINC00514 promotes lipogenesis and tumor progression in esophageal squamous cell carcinoma by sponging miR-378a-5p to enhance SPHK1 expression
}

\author{
XIN WANG $^{1 *}$, HONGTAO LIU ${ }^{2,3 *}$, QING ZHANG ${ }^{3}$, XUEYING ZHANG $^{2}$, YUE QIN $^{2}$, \\ GUANGZHAO ZHU ${ }^{2}$, JINGHAN DANG ${ }^{4}$, FENG WANG $^{5,6}$, XIANGXIANG YANG $^{1}$ and RUITAI FAN ${ }^{1}$
}

\begin{abstract}
${ }^{1}$ Department of Radiotherapy, The First Affiliated Hospital of Zhengzhou University, Zhengzhou, Henan 450052; ${ }^{2}$ School of Life Sciences, Zhengzhou University, Zhengzhou, Henan 450001; ${ }^{3}$ Translational Medicine Research Center, Zhengzhou People's Hospital, Zhengzhou, Henan 450003; ${ }^{4}$ Department of Clinical Medicine, Zhengzhou University, Zhengzhou, Henan 450052; ${ }^{5}$ Institute of Genomic Medicine, College of Pharmacy; ${ }^{6}$ International Cooperative Laboratory of Traditional Chinese Medicine Modernization and Innovative Drug Development of Chinese Ministry of Education, College of Pharmacy, Jinan University, Guangzhou, Guangdong 510632, P.R. China
\end{abstract}

Received July 6, 2021; Accepted August 23, 2021

DOI: 10.3892/ijo.2021.5266

\begin{abstract}
Increasing evidence has demonstrated that long non-coding RNAs serve pivotal roles in tumor development, progression, metastasis and metabolism. However, to the best of our knowledge, the roles and molecular mechanisms of long intergenic nonprotein-coding RNA 00514 (LINC00514) in esophageal squamous cell carcinoma (ESCC) remain unknown. The present study found that LINC00514 and sphingosine kinase 1 (SPHK1) were both upregulated in ESCC tissues and cells, and their high expression levels were closely associated with Tumor-Node-Metastasis stage, lymph node metastasis and poor prognosis of patients with ESCC. Functionally, knockdown of LINC00514 inhibited cell proliferation and invasion, and led to the downregulation of lipogenesis-related proteins, including SPHK1, fatty acid synthase, acetyl-coenzyme (Co)A carboxylase $\alpha$ and stearoyl-CoA desaturase 1, whereas LINC00514 overexpression promoted cell proliferation and invasion in ESCC KYSE150 and KYSE30 cells, and upregulated expression of lipogenesis-related proteins. Mechanistically, LINC00514 functioned as a competing endogenous RNA by sponging microRNA (miR)-378a-5p, resulting in the upregulation of SPHK1, which was accompanied by the activation of lipogenesis-related pathways, to promote ESCC
\end{abstract}

Correspondence to: Dr Ruitai Fan, Department of Radiotherapy, The First Affiliated Hospital of Zhengzhou University, 1 Jianshe East Road, Zhengzhou, Henan 450052, P.R. China

E-mail: fanruitai@126.com

*Contributed equally

Key words: long non-coding RNA, long intergenic nonprotein- coding RNA 00514, esophageal squamous cell carcinoma, microRNA-378a-5p, sphingosine kinase 1, lipogenesis cell proliferation and invasion. Taken together, these findings suggest that LINC00514 may participate in ESCC lipogenesis, and targeting the LINC00514/miR-378a-5p/SPHK1 signaling axis may be a novel and promising therapeutic strategy for management of patients with ESCC.

\section{Introduction}

Esophageal cancer (ESCA), as one of the most lethal malignant tumors of the digestive tract, exhibits rapid proliferative ability and early metastatic potential, and its incidence is gradually increasing worldwide $(1,2)$. ESCA presents as two primary histological types: i) Esophageal squamous cell carcinoma (ESCC) and; ii) esophageal adenocarcinoma (3). ESCC, the predominant histological type, accounts for $>80 \%$ of all cases of ESCA (4), particularly in the Henan province of China $(5,6)$. Despite significant progress in the molecular targeted therapy and other strategies of management, the 5-year survival rate of patients remains low $(15-25 \%)(7,8)$. Therefore, there remains an urgent need to further understand the pathogenesis of ESCC and to identify early biomarkers for the diagnosis and therapy of ESCC, which will provide novel opportunities for the treatment of patients with ESCC.

Long non-coding RNAs (lncRNAs) are a class of transcripts $>200$ nucleotides in length without protein coding potential (9). Increasing evidence has demonstrated that lncRNAs are closely implicated in numerous tumor phenotypes through a variety of molecular mechanisms, including transcriptional regulation, epigenetic modification, microRNA (miRNA/miR) sponging and RNA decay (10-12). With the rapid development of sequencing and molecular biology technologies, multiple ESCC-related IncRNAs have been identified, including lncRNA H19 (13), cancer susceptibility $9(14,15)$ and fragile X mental retardation 1-antisense RNA 1 (16), which have been widely implicated in the development and progression of ESCC via various mechanisms. Current data suggest 
that lncRNAs may constitute novel, promising biomarkers and therapeutic targets for patients with ESCC.

Long intergenic non-protein coding RNA 514 (LINC00514) is localized at chromosome 16p13.3, and it is 3,221-bp long (17). LINC00514 has been reported to be closely associated with the development and progression of tumors, including osteosarcoma (18), papillary thyroid cancer (19) and neuroendocrine prostate cancer (20). Sphingosine kinase 1 (SPHK1) is localized at chromosome 17q25.1, and it has been shown to participate in lipogenesis and tumor progression in a variety of tumors (21-23). However, to the best of our knowledge, the roles and molecular mechanisms of LINC00514 in ESCC remain to be investigated.

The present study investigated the expression and correlation of LINC00514 and SPHK1 in ESCC tissues and cells, and determined the clinical value of LINC000514 and SPHK1 in Tumor-Node-Metastasis (TNM) stage, lymph node metastasis and prognosis of patients with ESCC. Functionally, the roles of LINC00514 in cell proliferation, invasion and lipogenesis were explored. Mechanistically, the present study demonstrated the competing endogenous (ceRNA)-mediated mechanism of LINC00514, through its ability to absorb miR-378a-5p to promote SPHK1 expression and lipogenesis in ESCC cells, further promoting the proliferation and invasion of these cells. Taken together, the present data highlighted the role of LINC00514 in ESCC proliferation, invasion and lipogenesis, and identified a novel lipogenesis-related pathway based on LINC00514 and LINC00514/miR-378a-5p/SHPK1 signaling in ESCC cells. Therefore, the LINC00514/miR-378a-5p/SHPK1 signaling axis identified in the current study may be a novel and promising therapeutic target for patients with ESCC.

\section{Materials and methods}

Tissue samples. A total of 85 ESCC and corresponding normal tissues were obtained from The First Affiliated Hospital of Zhengzhou University (Zhengzhou, China).

The age range of all the patients was 41-85 years, and the median age was 64 years old. The clinicopathological features of the patients with ESCC enrolled in the present study were as follows: i) Sex: Male, 55 cases vs. female, 30 cases; ii) age: $<60$ years old, 38 cases vs. $\geq 60$ years old, 47 cases; iii) smoking history: Smoker, 41 cases vs. non-smoker, 44 cases; iv) alcohol consumption: Drinker, 49 cases vs. non-drinker, 36 cases; v) tumor diameter, $<4 \mathrm{~cm}, 51$ cases vs. $\geq 4 \mathrm{~cm}, 34$ cases; differentiation degree: High/moderate differentiation, 52 cases vs. poor differentiation, 33 cases; vi) TNM stage (24): I-II, 55 cases vs. III-IV, 30 cases; and vii) metastasis status: Lymph node metastasis, 28 cases vs. absence of lymph node metastasis, 57 cases.

Tumor tissue samples were confirmed as ESCC using hematoxylin and eosin staining by experienced pathologists at The First Affiliated Hospital of Zhengzhou University as routine. Informed consent for the use of all samples in the present study was obtained from each patient, and the study was approved by the Research and Ethics Committee of The First Affiliated Hospital of Zhengzhou University.

Public database analysis. starBase version 3.0 online software based on The Cancer Genome Atlas (TCGA) database was used to investigate the expression levels of LINC00514,
SPHK1 and miR-378a-5p in patients with ESCA and healthy individuals (starbase.sysu.edu.cn/panCancer.php), and the P-value of differential expression was directly obtained from the website. Gene Expression Profiling Interactive Analysis (GEPIA) was employed to determine SPHK1 expression. The Gene Expression Omnibus (GEO) dataset, GSE111011, was used to assess the expression of SPHK1 in 7 cases of ESCC and paired normal samples, whereas the GSE43732 was used to analyze the expression of miR-378a-5p in 119 cases of ESCC and paired normal samples. LncBase Predicted v.2 (carolina.imis.athena-innovation.gr/diana_ tools/web/index.php?r=lncbasev2/index-predicted) (25) was used to predict the binding site of LINC00514 and miR-378a-5p. TargetScan (targetscan.org/vert_71/) and miRDB (mirdb.org/) were employed to predict the downstream target genes of miR-378a-5p.

Cell lines and culture. Human ESCC cell lines (Eca109, KYSE150, KYSE30, KYSE450 and KYSE70) and normal esophageal epithelial Het-1A cells were obtained from The Cell Bank of Type Culture Collection of The Chinese Academy of Sciences, and were maintained in RPMI-1640 medium supplemented with 10\% FBS (Gibco; Invitrogen; Thermo Fisher Scientific, Inc.) in a humidified incubator with $5 \% \mathrm{CO}_{2}$. All cells were routinely tested and verified as negative for mycoplasma.

Cell transfection. LINC00514 small interfering RNA (siRNA)s 1, 2 and 3 were designed and chemically synthesized by Guangzhou RiboBio Co., Ltd. miR-378-5p mimic, negative control (NC) mimic, miR-378a-5p inhibitor and NC inhibitor were purchased from Shanghai GenePharma Co., Ltd. SPHK1 siRNA was purchased from Santa Cruz Biotechnology, Inc. pcDNA3.1 and pcDNA3.1-LINC00514/SPHK1 were constructed by TsingKe Biological Technology.

The aforementioned constructs were transfected into KYSE150 and KYSE30 cells using Lipofectamine ${ }^{\circledR} 2000$ (Invitrogen; Thermo Fisher Scientific, Inc.) according to the manufacturer's protocol.

Cell Counting Kit-8 (CCK-8) assay. The proliferation of ESCC KYSE150 and KYSE30 cells was assayed in triplicate according to the manufacturer's protocol. Briefly, KYSE150 and KYSE30 cells $\left(\sim 2 \times 10^{3}\right.$ cells/well) were seeded into 96-well plates. At the time of measurement, CCK-8 reagent (Beyotime Institute of Biotechnology) was added to the wells, and the absorbance value at $450 \mathrm{~nm}$ was determined using a microplate reader (Thermo Fisher Scientific, Inc.) to evaluate the proliferative ability of KYSE150 and KYSE30 cells.

Transwell assay. Cell invasion was determined using Transwell chambers coated with Matrigel (BD Biosciences). Briefly, the transfected KYSE150 and KYSE30 cells ( $1 \times 10^{5}$ cells) were seeded in the upper layer of the chamber, whereas the bottom chamber contained $20 \% \mathrm{FBS}$. A total of $48 \mathrm{~h}$ after seeding, the cells that had invaded were fixed using methanol for $30 \mathrm{~min}$ at room temperature, and then stained with $0.1 \%$ of crystal violet for $15 \mathrm{~min}$ at room temperature. Finally, the number of invasive cells was determined using a light microscope (Leica Microsystems, Inc.) (magnification, x200). 
Reverse transcription-quantitative PCR (RT-qPCR). Total RNA was isolated using TRIzol ${ }^{\circledR}$ (Invitrogen; Thermo Fisher Scientific, Inc.) according to the manufacturer's instructions. For mRNA analysis, RT-qPCR was performed using a Quant One Step qRT-PCR kit (SYBR Green; cat. no. FP303; Tiangen Biotech Co., Ltd.) on an ABI 7500 series PCR system (Applied Biosystems; Thermo Fisher Scientific, Inc.) using the following specific primers: LINC00514 forward 5'-CATCCAGATTTG GGCCCCTT-3' and reverse, 5'-CATGCCTGACCACGAATC CT-3' (product length, $231 \mathrm{bp}$ ); SPHK1 forward, 5'-CAGTGG TCGGTTGCGGAC-3' and reverse, 5'-GCACGTGACTCC GGAAGAG-3' (product length, $150 \mathrm{bp}$ ); and GAPDH forward, 5'-TCATCATCTCTGCCCCCTCT-3' and reverse 5'-GAT GGCATGGACTGTGGTCA-3' (product length, 188 bp). The thermocycling conditions were: $95^{\circ} \mathrm{C} 30 \mathrm{sec}$; followed by 30 cycles of $95^{\circ} \mathrm{C}$ for $5 \mathrm{sec}, 50^{\circ} \mathrm{C}$ for $30 \mathrm{sec}$ and $72^{\circ} \mathrm{C}$ for $30 \mathrm{sec}$. The results were normalized to GAPDH using the $2^{-\Delta \Delta \mathrm{Cq}}$ method (26).

For miR-378a-5p analysis, total RNA was reverse transcribed using a miRcute Plus miRNA First-Strand cDNA kit according to the manufacturer's protocol (Tiangen Biotech Co., Ltd.). qPCR amplification for miR-378a-5p was performed using the miRcute Plus miRNA qPCR kit (SYBR Green) (Tiangen Biotech Co., Ltd.) and the following specific forward primers along with the reverse primers from the kit: miR-378a-5p forward, 5'-CTCCTGACTCCAGGTCCTG TGT-3' and U6 forward, 5'-CTCGCTTCGGCAGCACA-3'. The thermocycling conditions were: $95^{\circ} \mathrm{C}$ for $30 \mathrm{sec}$; followed by 40 cycles of $95^{\circ} \mathrm{C}$ for $5 \mathrm{sec}$ and $60^{\circ} \mathrm{C}$ for $30 \mathrm{sec}$. The results were normalized to GAPDH using the $2^{-\Delta \Delta \mathrm{Cq}}$ method.

Subcellular fractionation. A Cell Nucleus and Cytoplasm RNA Isolation kit (Shaanxi Yuan Beibei Biological Technology Co., Ltd.) was used to extract the nuclear and cytoplasmic RNA according to the manufacturer's instructions. Briefly, KYSE150 and KYSE30 cells ( $3 \times 10^{7}$ cells) were harvested by centrifugation at $2,000 \times \mathrm{g}$ for $10 \mathrm{~min}$ at room temperature, and washed with ice-cold PBS. Cells were then resuspended in $300 \mu \mathrm{l} \mathrm{PBS}$ and centrifuged at $3,000 \mathrm{x} \mathrm{g}$ for $10 \mathrm{~min}$ at $4^{\circ} \mathrm{C}$. The supernatant was obtained as the cytoplasmic fraction. The precipitate was collected and resuspended in $300 \mu \mathrm{l}$ PBS and then centrifuged at $3,000 \mathrm{x}$ for $10 \mathrm{~min}$ at $4^{\circ} \mathrm{C}$. The precipitate was collected as the nuclear fraction.

Total RNA was isolated using TRIzol ${ }^{\circledR}$. Finally, the cytoplasmic and nuclear RNA was reverse transcribed to cDNA using a PrimeScript ${ }^{\mathrm{TM}}$ RT kit with gDNA Eraser (cat. no. RR047A; Takara Bio, Inc.) according to the manufacturer's protocol, were further investigated using RT-qPCR utilizing LINC00514, U6 and GAPDH-specific primers as described above. GAPDH and U6 were used as cytoplasmic and nuclear controls, respectively.

Fluorescence in situ hybridization (FISH) assay. LINC00514 probe (NR_033861.1, 5'-AACGGACCGGGAACCCAGCCA GGTCGGGGCCGAAGGGGCTGGGGTGGCTGGGGGAG A-3') was synthesized and labeled using cyanine 3 (Cy3) by Shanghai GenePharma Co., Ltd. FISH assays were performed using an RNA FISH kit (Shanghai GenePharma, Co., Ltd.) according to manufacturer's instructions. Briefly, KYSE150 and KYSE30 cells were grown in 24-well plates with glass cover slips for $24 \mathrm{~h}$. After immobilization using $4 \%$ paraformaldehyde for $15 \mathrm{~min}$ at room temperature and permeabilization using Triton X-100 for $15 \mathrm{~min}$ at room temperature, KYSE150 and KYSE30 cells were hybridized with $20 \mu \mathrm{M}$ Cy3-labeled LINC00514 probe, and DAPI was used to stain the nuclei of KYSE150 and KYSE30 cells for $10 \mathrm{~min}$ in dark at room temperature. Images were observed using a fluorescence microscope (magnification, x200).

Dual luciferase reporter assay. Dual luciferase reporter assays were performed to determine the interaction of miR-378a-5p with LINC00514 or SPHK1 in KYSE150 and KYSE30 cells. Recombinant vectors, pmirGLO-LINC00514-wild type (WT) and pmirGLO-LINC00514-mutant (MUT), as well as pmirGLO-SPHK1-WT and pmirGLO-SPHK1-MUT (all from TsingKe Biological Technology) along with miR-378a-5p mimic and NC mimic were co-transfected into KYSE150 and KYSE30 cells using Lipofectamine ${ }^{\circledR}$ 2000. Luciferase activity was determined using the Dual-Luciferase Reporter assay system (Promega Corporation) $48 \mathrm{~h}$ after transfection according to the manufacturer's protocol.

RNA immunoprecipitation (RIP) assay. RIP assay was performed in KYSE150 and KYSE30 cells using an RNA-Binding Protein Immunoprecipitation kit (MilliporeSigma) according to the manufacturer's protocol. Briefly, RIP lysates were prepared from KYSE150 and KYSE30 cells transfected with miR-378a-5p mimic or NC mimic using a Magna RIP ${ }^{\mathrm{TM}}$ RNA-binding Protein Immunoprecipitation kit (cat. no. 17-700; MilliporeSigma) by centrifugation at $1,000 \mathrm{x} \mathrm{g}$ for $5 \mathrm{~min}$ at $4^{\circ} \mathrm{C}$. Subsequently, $100 \mu \mathrm{l}$ lysate was subjected to IP using $5 \mu 1$ normal mouse IgG or $5 \mu \mathrm{l}$ anti-argonaute (Ago)2 antibody in the kit. The presence of LINC00514 or SPHK1 and miR-378a-5p enriched on beads was determined using RT-qPCR with the corresponding specific primers.

Western blotting. Total protein was extracted from ESCC cells using RIPA lysis buffer (Beijing Solarbio Science \& Technology Co., Ltd.), and the protein concentration was determined using a Bradford assay. The proteins (100 $\mu \mathrm{g} / \mathrm{lane})$ were loaded on $10 \%$ SDS-gels, resolved using SDS-PAGE and then transferred to PVDF membranes (MilliporeSigma). After blocking with skimmed milk for $2 \mathrm{~h}$ at room temperature, the membranes were incubated with primary antibodies against SPHK1 (cat. no. ab109522; 1:1,000), fatty acid synthetase (FASN) (cat. no. ab128870; 1:10,000), acetyl-CoA carboxylase $\alpha$ (ACACA) (cat. no. ab109368; 1:1,000), stearoyl-CoA desaturase 1 (SCD1) (cat. no. ab236868; 1:1,000) and $\beta$-actin (cat. no. ab115777; 1:200) (Abcam) overnight at room temperature. Subsequently, a secondary horseradish peroxidase-conjugated AffiniPure goat anti-rabbit IgG (AmyJet Scientific Inc.; cat. no. 111-035-003; 1:20,000) was added to the PVDF membranes. Finally, enhanced chemiluminescence reagent (Beyotime Institute of Biotechnology) was used to visualize the signals, and the quantification of the blots was analyzed using ImageJ version 1.8.0 (National Institutes of Health).

Statistical analysis. GraphPad Prism version 8.0 (GraphPad Software, Inc.) was used to analyze the experimental data, which are presented as the mean \pm standard deviation. The 
association between LINC00514, SPHK1, miR-378a-5p and the clinicopathological features were investigated using a $\chi^{2}$ test. Survival analysis was performed using a log-rank test. Spearman's rank correlation analysis was used to analyze the non-parametric data, and Pearson's correlation coefficient analysis was used to examine the parametric data. For the matched samples, the data was analyzed using a Wilcoxon signed rank test, and for non-matched samples, the data was compared using a Mann-Whitney U test. Comparisons between two groups were determined using a Student's t-test, whereas comparisons between $\geq 3$ groups were analyzed using a one-way ANOVA with a Dunnett's or Tukey's post hoc test to assess the difference between two groups. $\mathrm{P}<0.05$ was considered to indicate a statistically significant difference.

\section{Results}

Upregulated expression of LINC00514 in ESCC tissues is predictive of a poor prognosis. To explore the expression pattern of LINC00514 in ESCA tissues, starBase and RT-qPCR assays were used to determine the expression of LINC00514 in ESCA tissues. Data from starBase revealed that ESCA tissues exhibited higher expression levels of LINC00514 compared with those of normal samples ( $\mathrm{P}<0.05$; Fig. 1A). Furthermore, RT-qPCR analysis showed that the expression of LINC00514 in the 85 ESCC tissues was also higher compared with that of the paired normal samples ( $\mathrm{P}<0.0001$; Fig. 1B). Notably, patients with ESCC and high LINC00514 expression exhibited a lower survival rate than those with low LINC00514 expression (Fig. 1C).

In addition, it was found that LINC00514 was frequently upregulated in 13 other tumor types and downregulated in two other tumor types, including kidney chromophobe (KICH) and lung squamous cell carcinoma (Fig. S1). These data suggest that LINC00514 is closely associated with tumor development and progression in ESCC, and it may be a novel prognostic predictor for patients with ESCC.

Upregulated expression of SPHK1 in ESCC tissues. To analyze the expression pattern of SPHK1 in ESCA tissues, data from TCGA and GEO, as well as from the RT-qPCR analysis were used to determine the expression of SPHK1 in ESCA tissues. GEPIA online software showed that SPHK1 expression in ESCA tissues was significantly higher than that in normal tissues $(\mathrm{P}<0.05$; Fig. 1D), which was consistent with the data from starBase online software and the GEO dataset, GSE111011 (Fig. 1E and F).

Further analysis of the 85 cases of ESCC and corresponding normal tissues demonstrated that SPHK1 expression in ESCC tissues was markedly higher than that in paired normal tissues $(\mathrm{P}<0.0001$; Fig. $1 \mathrm{G})$, which was further validated in four randomly selected ESCC and paired normal tissues (Fig. 1H) as well as in a number of ESCC cell lines (Fig. 1I).

To further elucidate the correlations between LINC00514 and SPHK1, Pearson's correlation analysis was used, which revealed that LINC00514 expression exhibited a positive correlation with SPHK1 expression in the 85 ESCC tissues (Fig. 1J). Importantly, patients with ESCC who had a high SPHK1 expression level exhibited reduced survival rates compared with those of patients with ESCC who had low
SPHK1 expression (Fig. 1K). In addition, SPHK1 expression levels were higher in 13 other tumor types compared with those in the respective paired normal tissues, whereas it was only downregulated in KICH (Fig. S2). These findings suggest that SPHK1 may function as an oncogene in different tumor types, particularly in ESCC, and may be a novel prognostic predictor for patients with ESCC.

Association between LINCO0514 and SPHK1 expression levels with the clinicopathological features in patients with ESCC. To unveil the possible biological functions of LINC00514 and SPHK1 in ESCC, the present study further investigated the associations between the expression levels of LINC00514 and SPHK1 and the clinicopathological features. It was found that the expression of LINC00514 and SPHK1 was closely associated with TNM stage and lymph node metastasis in patients with ESCC, but was not associated with patients' sex, age, smoking history, alcohol consumption, tumor diameter or degree of differentiation (Tables I and II). These findings suggest that LINC00514 and SPHK1 may participate in the progression and metastasis of ESCC.

LINC00514 promotes the proliferation and invasion of ESCC cells by regulating lipogenesis-related proteins. In order to reveal the biological functions of LINC00514 in ESCC, RT-qPCR was used to detect the expression of LINC00514 in a number of ESCC cell lines. It was found that all ESCC cell lines exhibited higher levels of LINC00514 expression compared with the Het-1A cells, a normal esophageal epithelial cell line (all $\mathrm{P}<0.0001 ;$ Fig. $2 \mathrm{~A}$ ), which was consistent with the data derived from the ESCC tissues.

In addition, three siRNAs against LINC00514 markedly downregulated the expression of LINC00514 in KYSE150 and KYSE30 cells, which harbored the highest levels of LINC00514, and LINC00514 siRNA2 displayed the most effective interference efficacy in these cell lines (Fig. 2B). Functionally, knockdown of LINC00514 significantly suppressed the proliferation of KYSE150 and KYSE30 cells after 24, 48, 72 and $96 \mathrm{~h}(\mathrm{P}<0.05$; Fig. 2C), as well as their invasive ability after $48 \mathrm{~h}$ (Fig. 2D and E). In addition, knockdown of LINC00514 led to the downregulation of expression of lipogenesis-related proteins, including SPHK1, FASN, ACACA and SCD1 (Fig. 2F). By contrast, pcDNA3.1-LINC00514 significantly increased the expression of LINC00514 in KYSE150 and KYSE30 cells (Fig. 3A), significantly increased cell proliferation and invasion in KYSE150 and KYSE30 cells, and led to an increase in the protein expression levels of SPHK1, FASN, ACACA and SCD1 (Fig. 3B-E). These findings suggest that LINC00514 may function as an oncogene by affecting lipogenesis in ESCC cells.

LINC00514 affects miR-378a-5p expression by acting as a ceRNA. To further explore the possible molecular mechanisms of LINC00514 in ESCC cells, a localization assay was performed using RT-qPCR. It was found that LINC00514 was primarily localized in the cytoplasm of KYSE150 and KYSE30 cells (Fig. 4A), which was validated using a FISH assay (Fig. 4B), suggesting that LINC00514 functions via a ceRNA-mediated mechanism in ESCC cells. 


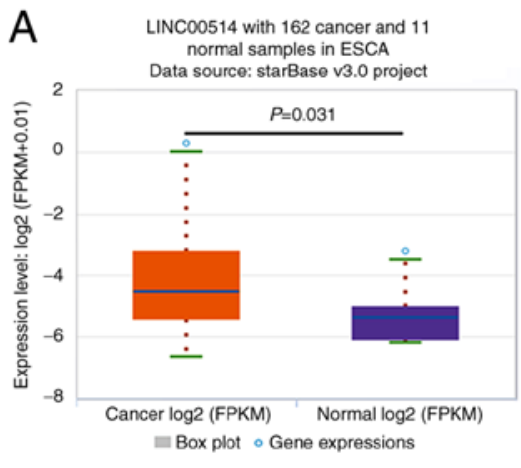

D

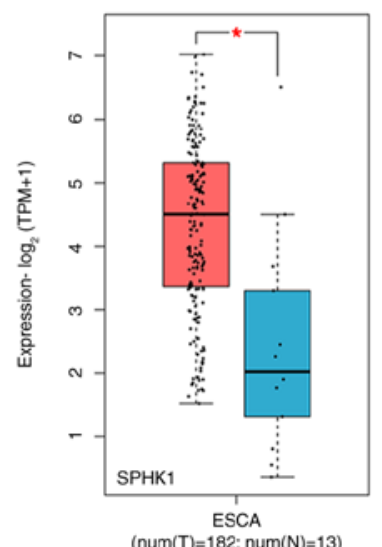

G

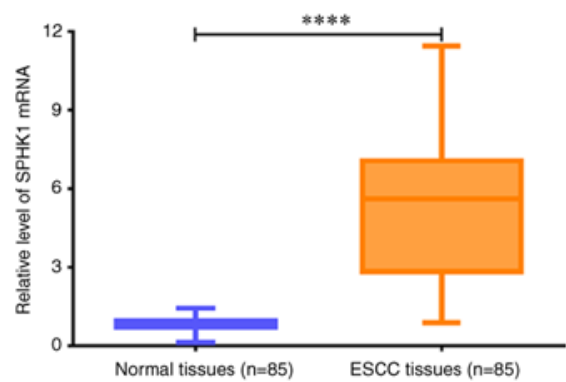

I

Het-1A Eca109 KYSE150 KYSE30 KYSE450 KYSE70

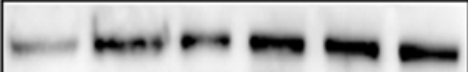

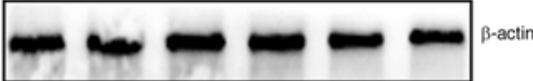

B

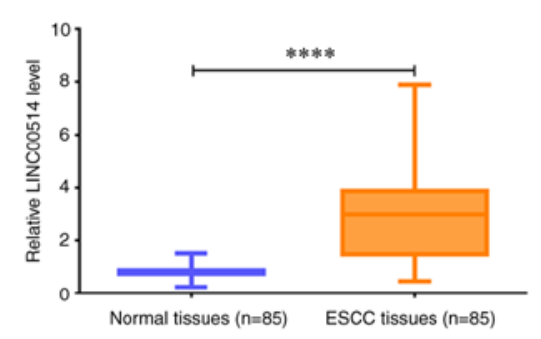

$\mathrm{E}$

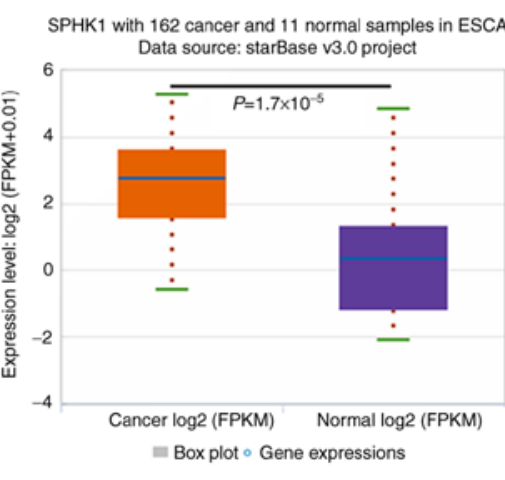

C

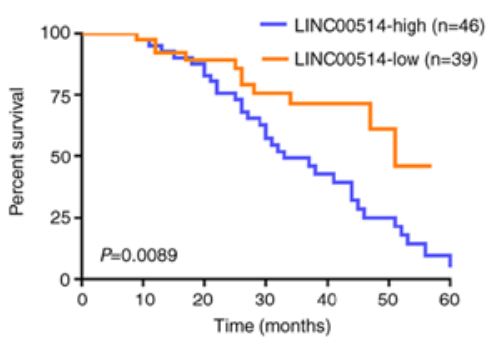

$\mathrm{F}$

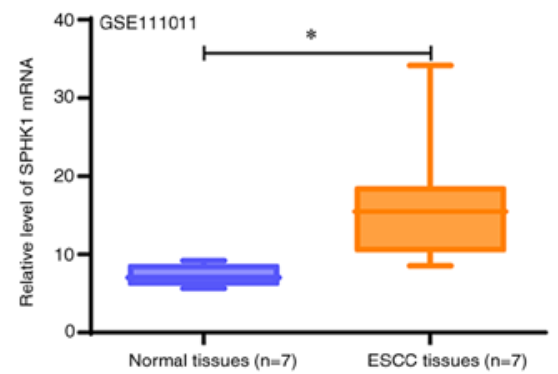

$\mathrm{H}$
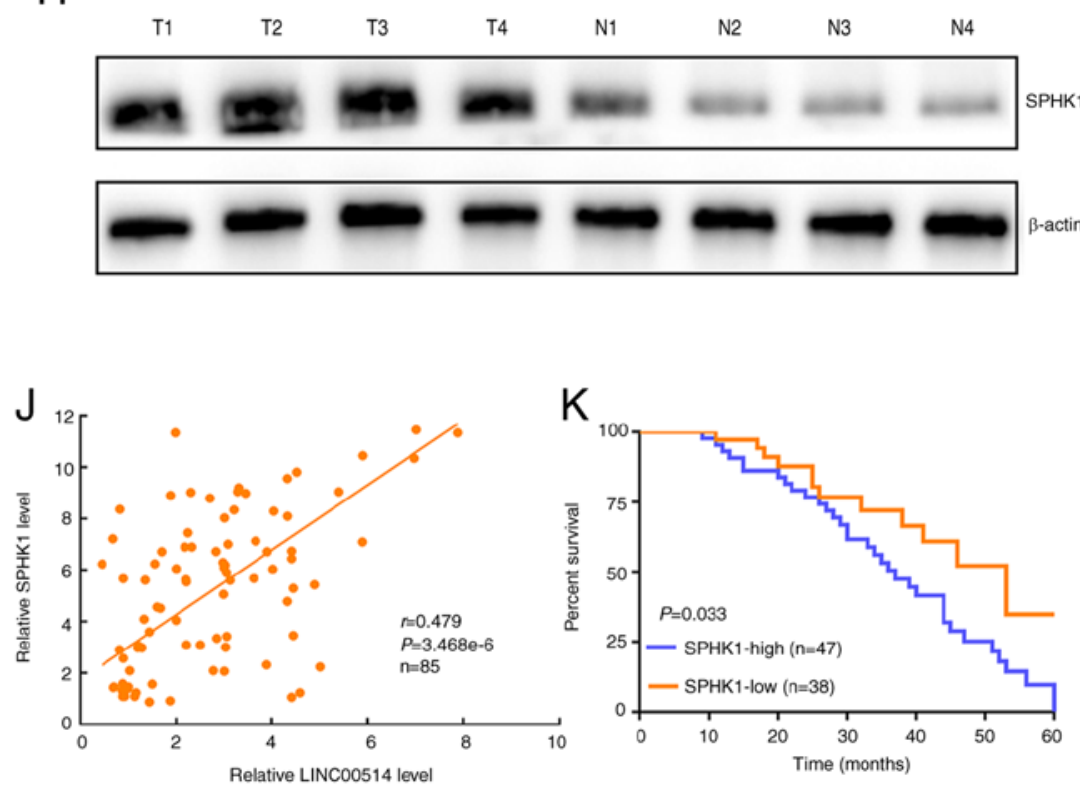

Figure 1. Expression and prognostic value of LINC00514 and SPHK1 in ESCC. (A) starBase was used to investigate the expression of LINC00514 in 162 esophageal cancer samples and 11 normal samples. (B) Reverse transcription-quantitative PCR was used to evaluate the expression levels of LINC00514 in 85 ESCC and paired normal samples using a Wilcoxon signed rank test. ${ }^{* * * *} \mathrm{P}<0.0001$. (C) A log-rank test was used to analyze the effects of LINC00514 expression on the survival rate of patients with ESCC. (D) Gene Expression Profiling Interactive Analysis online software analysis of SPHK1 level in 182 ESCA and 13 normal samples. (E) starBase analysis was performed to determine the expression of SPHK1 in 162 ESCA and 11 normal samples. (F) The GSE111011 Gene Expression Omnibus dataset was used to detect the levels of SPHK1 in seven ESCC and paired normal samples using paired a Student's t-test. ${ }^{*} \mathrm{P}<0.05$. (G) Reverse transcription-quantitative PCR assay was used to determine the SPHK1 levels in 85 ESCC and paired normal samples, and the data was analyzed using a Wilcoxon signed rank test. (H) SPHK1 protein expression in four randomly selected paired tissues. $\beta$-actin was used as the loading control. (I) SPHK1 protein expression in ESCC cell lines (Eca109, KYSE150, KYSE30, KYSE450 and KYSE70) and the normal esophageal epithelial cell line Het-1A. $\beta$-actin was used as a loading control. (J) Spearman's rank correlation analysis of the correlation between LINC00514 and SPHK1 expression in the 85 ESCC and paired normal samples. (K) Log-rank analysis of the effect of SPHK1 expression on the survival rate of patients with ESCC. ${ }^{* * * *} \mathrm{P}<0.0001$. LINC00514, long intergenic nonprotein-coding RNA 00514; ESCC, esophageal squamous cell carcinoma; SPHK1, sphingosine kinase 1; ESCA, esophageal cancer; T, tumor; N, normal.

Subsequently, LncBase Predicted v. 2 which is integrated into DIANA Tools was used to predict the possible miRNAs that bound to LINC00514, and it was found that LINC00514 harbored binding sites for miR-378a-5p (Fig. 4C). 
Table I. Associations between LINC00514 expression and the clinicopathological features of the patients with ESCC.

\begin{tabular}{|c|c|c|c|c|c|}
\hline \multirow[b]{2}{*}{ Features } & \multirow[b]{2}{*}{$\mathrm{n}$} & \multicolumn{2}{|c|}{$\begin{array}{c}\text { LINC00514 } \\
\text { expression }\end{array}$} & \multirow[b]{2}{*}{$\chi^{2}$} & \multirow[b]{2}{*}{ P-value } \\
\hline & & + & - & & \\
\hline Sex & & & & 2.172 & 0.141 \\
\hline Male & 55 & 33 & 22 & & \\
\hline Female & 30 & 13 & 17 & & \\
\hline Age, years & & & & 1.261 & 0.262 \\
\hline$<60$ & 38 & 18 & 20 & & \\
\hline$\geq 60$ & 47 & 28 & 19 & & \\
\hline Smoking & & & & 1.500 & 0.221 \\
\hline Yes & 41 & 25 & 16 & & \\
\hline No & 44 & 21 & 23 & & \\
\hline Drinking & & & & 1.196 & 0.274 \\
\hline Yes & 49 & 29 & 20 & & \\
\hline No & 36 & 17 & 19 & & \\
\hline Tumor diameter, $\mathrm{cm}$ & & & & 1.335 & 0.248 \\
\hline$<4$ & 51 & 25 & 26 & & \\
\hline$\geq 4$ & 34 & 21 & 13 & & \\
\hline Differentiation degree & & & & 1.968 & 0.161 \\
\hline High/moderate & 52 & 25 & 27 & & \\
\hline Poor & 33 & 21 & 12 & & \\
\hline Tumor-Node-Metastasis stage & & & & 4.710 & $0.030^{\circ}$ \\
\hline I-II & 55 & 25 & 30 & & \\
\hline III-IV & 30 & 21 & 9 & & \\
\hline Lymph node metastasis & & & & 5.039 & $0.025^{\circ}$ \\
\hline Yes & 28 & 20 & 8 & & \\
\hline No & 57 & 26 & 31 & & \\
\hline
\end{tabular}

${ }^{\mathrm{a}} \mathrm{P}<0.05$.

To further confirm the interaction of LINC00514 with miR-378a-5p, a dual-luciferase reporter assay was conducted to investigate the binding of LINC00514 with miR-378a-5p. It was found that the luciferase intensity was markedly reduced by co-transfecting miR-378a-5p mimic and pmirGLO-LINC00514-WT vector into KYSE150 and KYSE30 cells, but not by co-transfecting miR-378a-5p mimic and pmirGLO-LINC00514-MUT vector (which lacks the miR-378-5p binding site) (Fig. 4D).

To further validate this result, an Ago2-RIP assay was performed, which revealed that endogenous LINC00514 and miR-378a-5p were preferentially enriched in Ago2-RIP, compared with the findings in control IgG-RIP (Fig. 4E and F). Importantly, LINC00514 enrichment was much higher in the miR-378a-5p mimic group than that in the NC mimic group $(\mathrm{P}<0.0001$; Fig. $4 \mathrm{G})$, suggesting that LINC00514 and miR-378a-5p appeared in the same RNA-induced silencing complex.

To verify the effects of LINC00514 knockdown or overexpression on the expression of miR-378a-5p in ESCC cells, RT-qPCR was performed, and it was found that LINC00514 depletion notably enhanced the expression of miR-378a-5p $(\mathrm{P}<0.0001$; Fig. 4H), whereas LINC00514 overexpression markedly suppressed the expression of miR-378a-5p $(\mathrm{P}<0.0001$; Fig. 4I). These findings suggest that LINC00514 directly affects the expression of miR-378a-5p by acting as a ceRNA in ESCC cells.

Associations between miR-378a-5p expression and clinicopathological features of patients with ESCC. Considering the interaction of LINC00514 with miR-378a-5p in ESCC cells, whether miR-378a-5p participated in the development and progression of ESCC was next investigated. miR-378a-5p was closely associated with TNM stage and lymph node metastasis in patients with ESCC $(\mathrm{P}<0.01)$, but was not associated with patients' sex, age, smoking habits, drinking habits, tumor diameter or degree of differentiation (Table III). These findings suggest that miR-378a-5p may participate in the progression and metastasis of ESCC.

SPHK1 is the direct target of miR-378a-5p in ESCC cells. To further investigate the downstream molecular mechanisms of 
Table II. Associations of SPHK1 expression with the clinicopathological features of patients with ESCC.

\begin{tabular}{|c|c|c|c|c|c|}
\hline \multirow[b]{2}{*}{ Features } & \multirow[b]{2}{*}{$\mathrm{n}$} & \multicolumn{2}{|c|}{ SPHK1 expression } & \multirow[b]{2}{*}{$\chi^{2}$} & \multirow[b]{2}{*}{ P-value } \\
\hline & & + & - & & \\
\hline Sex & & & & 0.526 & 0.468 \\
\hline Male & 55 & 32 & 23 & & \\
\hline Female & 30 & 15 & 15 & & \\
\hline Age, years & & & & 1.746 & 0.186 \\
\hline$<60$ & 38 & 18 & 20 & & \\
\hline$\geq 60$ & 47 & 29 & 18 & & \\
\hline Smoking & & & & 0.337 & 0.562 \\
\hline Yes & 41 & 24 & 17 & & \\
\hline No & 44 & 23 & 21 & & \\
\hline Drinking & & & & 1.646 & 0.200 \\
\hline Yes & 49 & 30 & 19 & & \\
\hline No & 36 & 17 & 19 & & \\
\hline Tumor diameter, $\mathrm{cm}$ & & & & 2.031 & 0.154 \\
\hline$<4$ & 51 & 25 & 26 & & \\
\hline$\geq 4$ & 34 & 22 & 12 & & \\
\hline Differentiation degree & & & & 0.616 & 0.433 \\
\hline High/moderate & 52 & 27 & 25 & & \\
\hline Poor & 33 & 20 & 13 & & \\
\hline Tumor-Node-Metastases stage & & & & 8.567 & $0.003^{\circ}$ \\
\hline I-II & 55 & 24 & 31 & & \\
\hline III-IV & 30 & 23 & 7 & & \\
\hline Lymph node metastasis & & & & 6.559 & $0.010^{2}$ \\
\hline Yes & 28 & 21 & 7 & & \\
\hline No & 57 & 26 & 31 & & \\
\hline
\end{tabular}

${ }^{\mathrm{a}} \mathrm{P} \leq 0.01$.

miR-378a-5p in ESCC cells, the expression of miR-378a-5p in ESCC tissues was analyzed. The data derived from TCGA and the GEO datasets revealed that miR-378a-5p expression was markedly reduced in ESCA (Fig. 5A) and ESCC tissues (Fig. 5B), which was validated by RT-qPCR in 85 cases of ESCC and paired normal tissues (Fig. 5C). In addition, the miR-378a-5p levels in a number of ESCC cell lines (Eca109, KYSE150, KYSE30, KYSE450 and KYSE70) was significantly lower than that in the normal esophageal epithelial cell line Het-1A (all $\mathrm{P}<0.0001$; Fig. 5D).

Notably, patients with ESCC and high miR-378a-5p levels exhibited improved survival rates compared with patients with ESCC and low miR-378a-5p levels (Fig. 5E). Bioinformatics analysis showed that miR-378a-5p exhibited lower levels in another 11 different tumor types compared with those in the corresponding paired normal tissues (Fig. S3). These data suggest that miR-378a-5p may function as a tumor suppressor in multiple tumor types.

TargetScan and miRDB online software were used to predict the possible downstream target genes of miR-378a-5p, and it was found that SPHK1 may be the potential target of miR-378a-5p (Fig. 6A). To validate this predicted result, a dual-luciferase reporter assay was performed, which showed that luciferase activity was markedly reduced in KYSE150 and KYSE30 cells co-transfected with miR-378a-5p mimic and pmirGLO-SPHK1-WT vector, whereas this was not observed with the pmirGLO-SPHK1-MUT vector, which lacks the miR-378-5p binding site (Fig. 6B).

To further validate this result, an Ago2-RIP assay was performed, which revealed that SPHK1 was markedly enriched in the Ago2-RIP compared with that observed in the control IgG-RIP (Fig. 6C). Notably, SPHK1 enrichment was much higher in the miR-378a-5p mimic group than that in the NC mimic group $(\mathrm{P}<0.0001$; Fig. 6D).

Further evaluation demonstrated that miR-378a-5p expression exhibited a negative correlation with SHPK1 expression in 85 ESCC tissues compared with their paired normal tissues (Fig. 6E). In addition, miR-378a-5p mimic significantly promoted the expression of miR-378a-5p in KYSE150 and KYSE30 cells (Fig. 6F), whereas miR-378a-5p inhibitor markedly suppressed the expression of miR-378a-5p in KYSE150 and KYSE30 cells (Fig. 6G).

Finally, it was found that the miR-378a-5p mimic significantly downregulated the expression of SPHK1 in KYSE150 

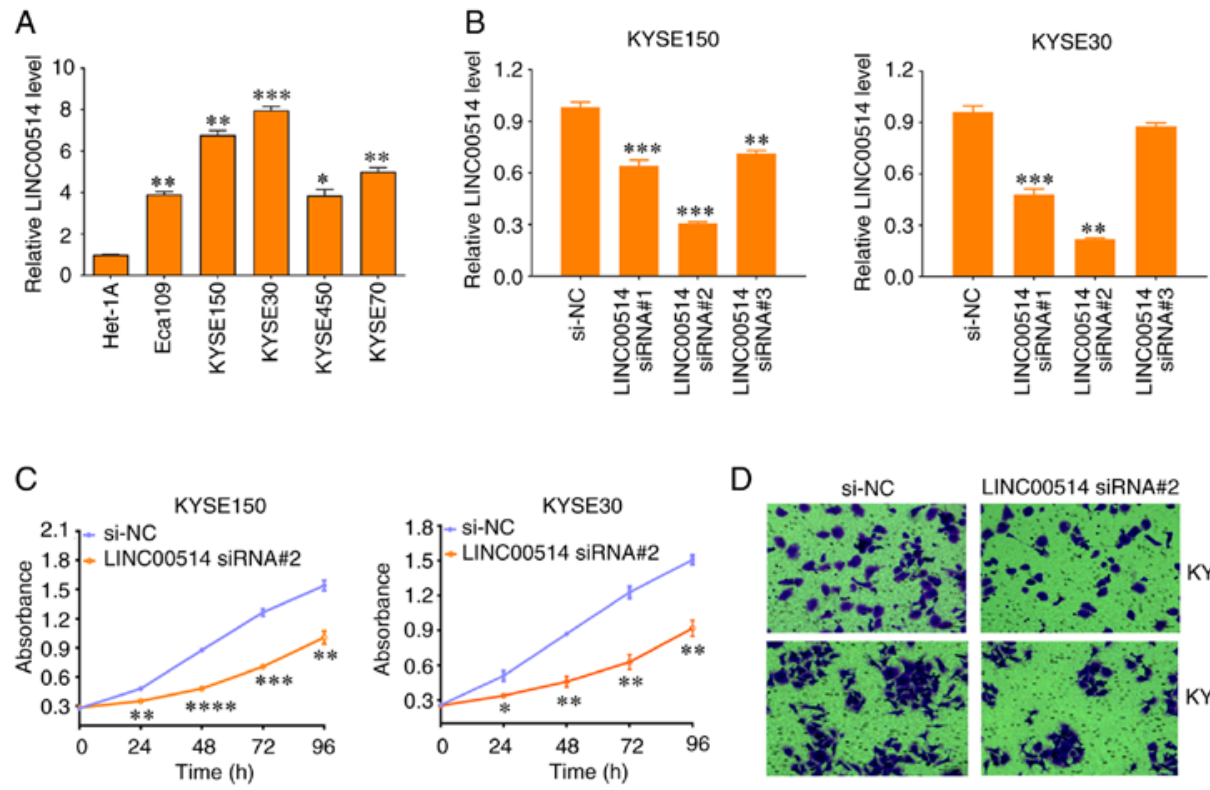

LINC00514 SIRNA\#2

$\mathrm{E}$
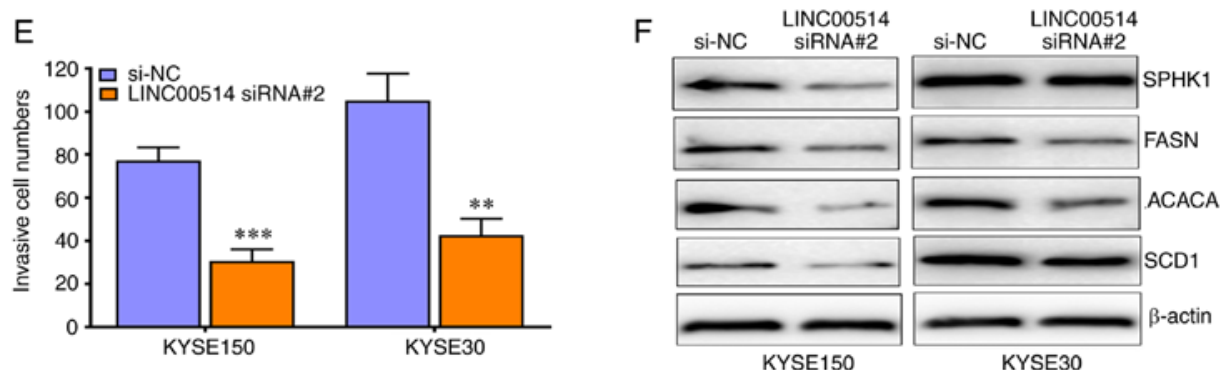

Figure 2. LINC00514 Knockdown suppresses the proliferation, invasion and expression of lipogenesis-related proteins in ESCC cells. (A) Reverse transcription-quantitative PCR assay of LINC00514 expression in various ESCC cell lines, including Eca109, KYSE150, KYSE30, KYSE450 and KYSE70 as well as in the normal esophageal epithelial Het-1A cell line. Data were compared using an ANOVA followed by a post hoc Dunnett's test. ${ }^{*} \mathrm{P}<0.05$, ${ }^{* * *} \mathrm{P}<0.01,{ }^{* * * *} \mathrm{P}<0.001$ vs. Het-1A. (B) Three siRNAs specific for LINC00514 significantly downregulated the expression of LINC00514 in KYSE150 and KYSE30 cells. Data were compared using an ANOVA followed by a post hoc Dunnett's test. (C-E) LINC00514 siRNA2 suppressed the proliferation and invasion of KYSE150 and KYSE30 cells. (F) LINC00514 siRNA2 reduced the expression of lipogenesis-related proteins (SPHK1, FASN, ACACA and SCD1) in KYSE150 and KYSE30 cells. ${ }^{*} \mathrm{P}<0.05$, ${ }^{* * *} \mathrm{P}<0.01,{ }^{* * * *} \mathrm{P}<0.001,{ }^{* * * *} \mathrm{P}<0.0001$ vs. si-NC group. LINC00514, long intergenic nonprotein-coding RNA 00514; ESCC, esophageal squamous cell carcinoma; SPHK1, sphingosine kinase 1; FASN, fatty acid synthetase; ACACA, acetyl-CoA carboxylase $\alpha$; SCD1, stearoyl-CoA desaturase 1; siRNA, small interfering RNA

A

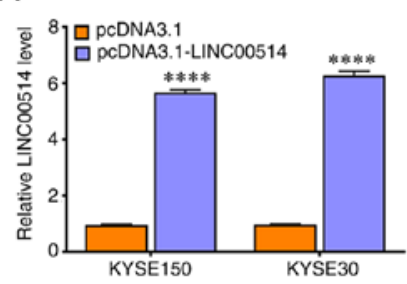

C

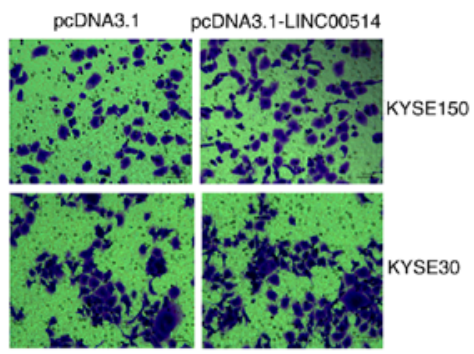

B

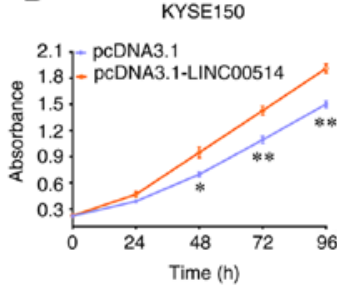

D

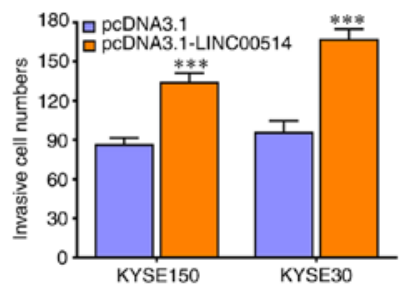

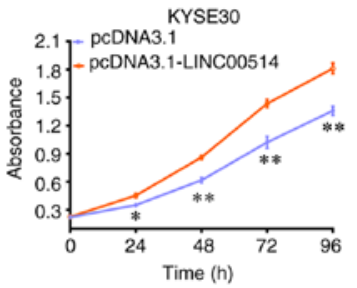

$\mathrm{E}$ pCDNA3.1 2 LINCONA3.1-

Figure 3. LINC00514 promotes the proliferation, invasion and expression of lipogenesis-related proteins in ESCC cells. (A) pcDNA3.1-LINC00514 transfection significantly upregulated the expression of LINC00514 in KYSE150 and KYSE30 cells. (B-D) LINC00514 overexpression promoted the proliferation and invasion of KYSE150 and KYSE30 cells. (E) LINC00514 overexpression promoted the expression of lipogenesis-related proteins (SPHK1, FASN, ACACA and SCD1) in KYSE150 and KYSE30 cells. ${ }^{*} \mathrm{P}<0.05,{ }^{* *} \mathrm{P}<0.01,{ }^{* * *} \mathrm{P}<0.001,{ }^{* * * *} \mathrm{P}<0.0001$. LINC00514, long intergenic nonprotein-coding RNA 00514; ESCC, esophageal squamous cell carcinoma; SPHK1, sphingosine kinase 1; FASN, fatty acid synthetase; ACACA, acetyl-CoA carboxylase $\alpha$; SCD1, stearoyl-CoA desaturase 1; siRNA, small interfering RNA. 
A

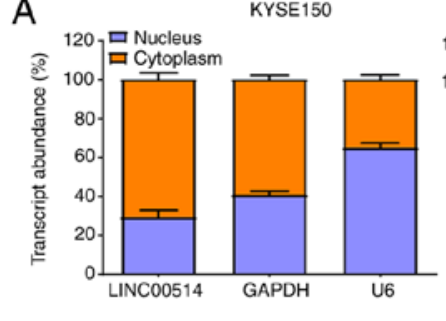

C

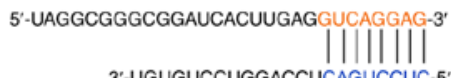

3'-UGUGUCCUGGACCUCAGUCCUC-5'

5'-UAGGCGGGCGGAUCACUUGAGCAGUCCUC-3'

D

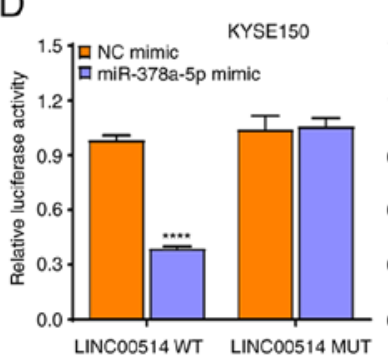

LINC00514 WT LINC00514 MUT

G

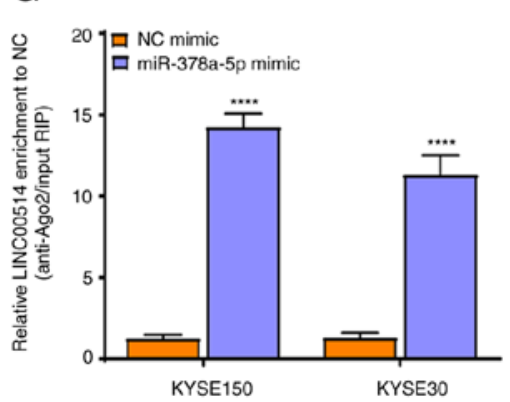

KYSE30

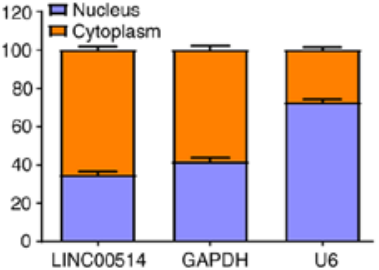

LINC00514-MUT

miR-378a-5p
B

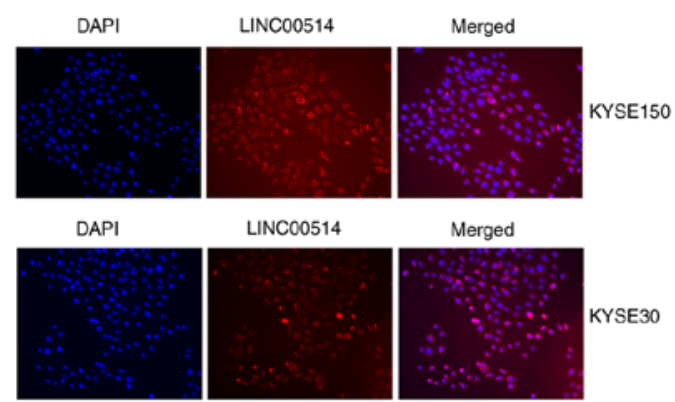

LINC00514-MUT
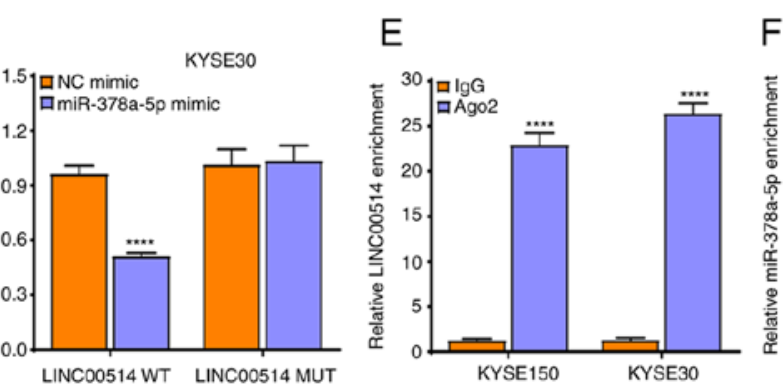

$\mathrm{F}$

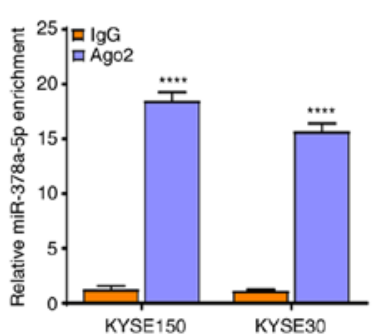

$\mathrm{H}$

I
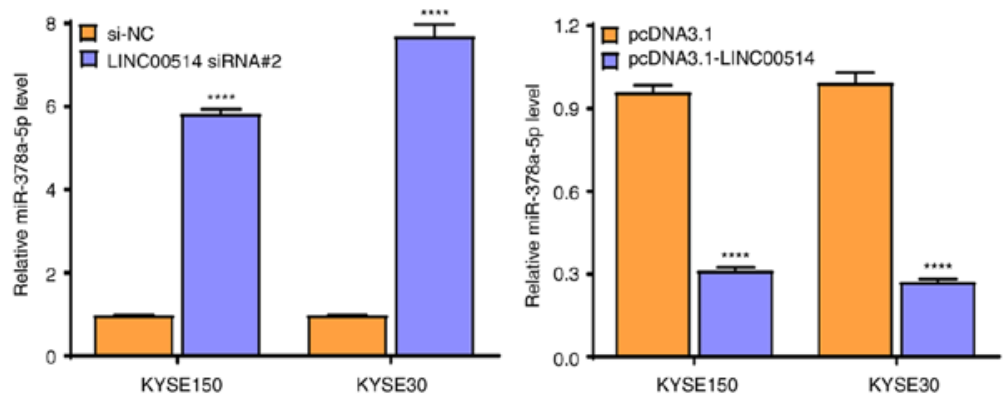

Figure 4. LINC00514 functions as a competing endogenous RNA by sponging miR-378a-5p in ESCC cells. (A) Reverse transcription-quantitative PCR was used to analyze the subcellular localization of LINC00514 in KYSE150 and KYSE30 cells. (B) Subcellular localization of LINC00514 in KYSE150 and KYSE30 cells was investigated using a fluorescence in situ hybridization assay, where LINC00514 was labeled by cyanine 3 (red) and the nuclei were stained with DAPI (blue). (C) lncBase was used to identify the association between LINC00514 and miR-378a-5p. (D) Dual-luciferase reporter assays were performed to investigate the binding between LINC00514 and miR-378a-5p in KYSE150 and KYSE30 cells. An Ago2-RIP assay was used to analyze the enrichment of (E) LINC00514 or (F) miR-378a-5p in the RISC in KYSE150 and KYSE30 cells. (G) Ago2-RIP assay was employed to analyze the enrichment of LINC00514 in KYSE150 and KYSE30 cells in the presence of miR-378a-5p mimic. (H) Transfection of LINC00514 siRNA 2 significantly promoted the expression of miR-378a-5p in KYSE150 and KYSE30 cells. (I) LINC00514 overexpression markedly suppressed the expression of miR-378a-5p in KYSE150 and KYSE30 cells. ${ }^{* * * * *} \mathrm{P}<0.0001$. LINC00514, long intergenic nonprotein-coding RNA 00514; ESCC, esophageal squamous cell carcinoma; miR, microRNA; Ago2, argonaute 2; RIP, RNA immunoprecipitation; RISC, RNA-induced silencing complex; siRNA, small interfering RNA.

and KYSE30 cells (Fig. 6H), whereas miR-378a-5p inhibitor increased the expression of SPHK1 in KYSE150 and KYSE30 cells (Fig. 6I). These data confirm that SPHK1 is a direct molecular target of miR-378a-5p in ESCC cells.

Roles of the LINC00514/miR-378a-5p/SPHK1 signaling axis in the proliferation and invasion of ESCC cells. To clarify the underlying roles and related molecular mechanisms of the LINC00514/miR-378a-5p/SPHK1 signaling axis in the proliferation and invasion of ESCC cells, KYSE150 and KYSE30 cells were transfected with miR-378a-5p mimic or inhibitor combined with pcDNA3.1-LINC00514, or pcDNA3.1-SPHK1 combined with LINC00514 siRNA2 or SPHK1 siRNA.

Next, CCK-8 and Transwell assays were used to examine the proliferation and invasion of the transfected cells. The results showed that miR-378a-5p mimic markedly suppressed the proliferation and invasion of KYSE150 and KYSE30 cells, which was partly reversed by pcDNA3.1-LINC00514 and pcDNA3.1-SPHK1 (Fig. 7A-C).

Importantly, miR-378a-5p mimic downregulated the expression of lipogenesis-related proteins, including SPHK1, FASN, ACACA and SCD1, which was also partly reversed by pcDNA3.1-LINC00514 and pcDNA3.1-SPHK1 (Fig. 7D). Conversely, miR-378a-5p inhibitor markedly promoted the proliferation and invasion of KYSE150 and KYSE30 cells, whereas LINC00514 siRNA2 and SPHK1 siRNA significantly reduced the promotive efficacy of miR-378a-5p inhibitor on the proliferation and invasion of KYSE150 and KYSE30 cells (Fig. 8A-C). miR-378a-5p inhibitor induced an upregulation in the protein expression levels of SPHK1, FASN, ACACA and SCD1, which was also in part reversed by LINC00514 siRNA2 and SPHK1 siRNA (Fig. 8D). 
Table III. Associations of miR-378a-5p expression with clinicopathological features of patients with ESCC.

\begin{tabular}{|c|c|c|c|c|c|}
\hline \multirow[b]{2}{*}{ Features } & \multirow[b]{2}{*}{$\mathrm{n}$} & \multicolumn{2}{|c|}{$\begin{array}{c}\text { LINC00514 } \\
\text { expression }\end{array}$} & \multirow[b]{2}{*}{$\chi^{2}$} & \multirow[b]{2}{*}{ P-value } \\
\hline & & + & - & & \\
\hline Sex & & & & 0.353 & 0.552 \\
\hline Male & 55 & 22 & 33 & & \\
\hline Female & 30 & 14 & 16 & & \\
\hline Age, years & & & & 0.708 & 0.400 \\
\hline$<60$ & 38 & 18 & 20 & & \\
\hline$\geq 60$ & 47 & 18 & 29 & & \\
\hline Smoking & & & & 1.079 & 0.299 \\
\hline Yes & 41 & 15 & 26 & & \\
\hline No & 44 & 21 & 23 & & \\
\hline Drinking & & & & 0.112 & 0.738 \\
\hline Yes & 49 & 20 & 29 & & \\
\hline No & 36 & 16 & 20 & & \\
\hline Tumor diameter, $\mathrm{cm}$ & & & & 2.321 & 0.128 \\
\hline$<4$ & 51 & 25 & 26 & & \\
\hline$\geq 4$ & 34 & 11 & 23 & & \\
\hline Differentiation degree & & & & 0.193 & 0.660 \\
\hline High/moderate & 52 & 23 & 29 & & \\
\hline Poor & 33 & 13 & 20 & & \\
\hline Tumor-Node-Metastasis stage & & & & 6.869 & $0.009^{\mathrm{a}}$ \\
\hline I-II & 55 & 29 & 26 & & \\
\hline III-IV & 30 & 7 & 23 & & \\
\hline Lymph node metastasis & & & & 7.488 & $0.006^{\mathrm{a}}$ \\
\hline Yes & 28 & 6 & 22 & & \\
\hline No & 57 & 30 & 27 & & \\
\hline
\end{tabular}

${ }^{\mathrm{a}} \mathrm{P} \leq 0.01$.

Overall, these data suggest that the LINC00514/ miR-378a-5p/SPHK1 signaling axis may play a pivotal role in ESCC lipogenesis, and manipulating this signaling axis may be a novel therapeutic strategy for patients with ESCC (Fig. 9).

\section{Discussion}

An increasing number of studies have revealed that numerous lncRNAs are widely implicated in tumor initiation, development, progression and metastasis $(21,27,28)$. IncRNAs have been regarded as excellent candidates for biomarkers and therapeutic targets in a large number of tumor types (29-33). Multiple lncRNAs exhibited differential expression in numerous different human tumors, and were confirmed to harbor diagnostic and prognostic potential (34-37). LINC00514 was reported to be involved in the development and progression of neuroendocrine prostate cancer. Mi et al (38) found that the expression of LINC00514 was markedly upregulated in osteosarcoma tissues and cells, and high levels of LINC00514 were positively associated with advanced tumor stages, distant metastasis and reduced overall survival of patients. Another study on osteosarcoma confirmed that LINC00514 was upregulated in osteosarcoma tissues and cells, and an increased LINC00514 level was associated with tumor size, TNM stage and distant metastasis. Furthermore, patients with osteosarcoma and high LINC00514 levels had a shorter overall survival rate (18).

Despite the fact that the role of LINC00514 has been investigated in various tumor types, its clinical value and possible biological functions remain to be identified in ESCC, to the best of our knowledge. The current study found that LINC00514 exhibited high expression levels in ESCC tissues and cell lines, and this high expression was closely associated with TNM stage, lymph node metastasis and reduced survival rates in patients with ESCC. These data highlighted the important clinical value of LINC00514 in ESCC.

Several studies demonstrated that LINC00514 was implicated in the regulation of cell proliferation and metastasis of different tumors. Yu et al (18) verified that LINC00514 knockdown suppressed cell proliferation in vitro and in vivo, as well as colony formation, migration and invasion 
A hsa-miR-378a-5p with 162 cancer and 11

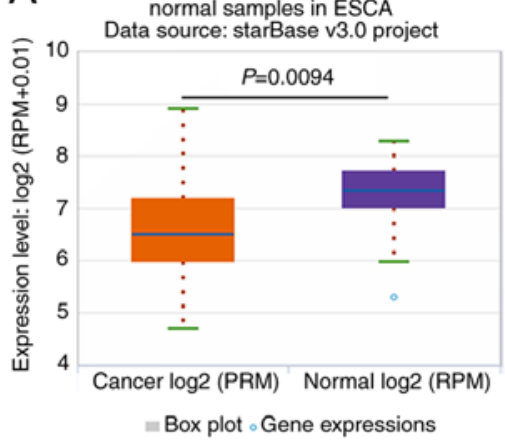

$\mathrm{D}$

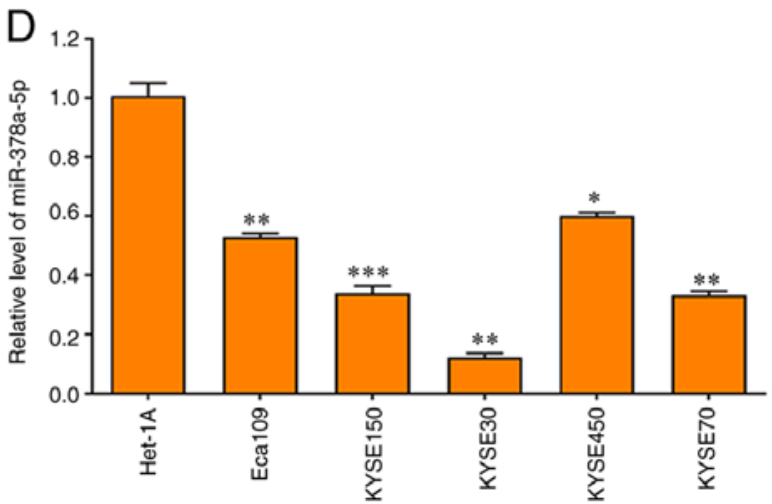

B

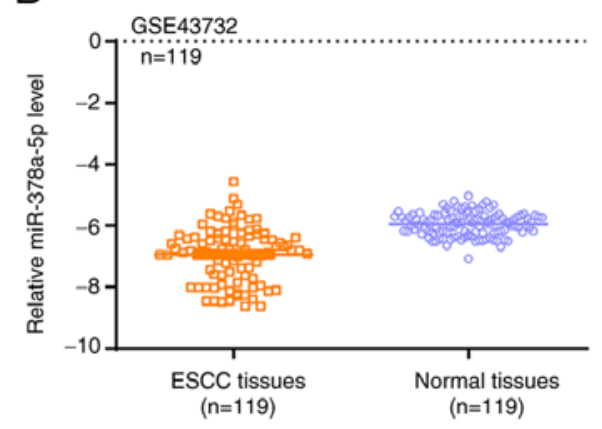

C

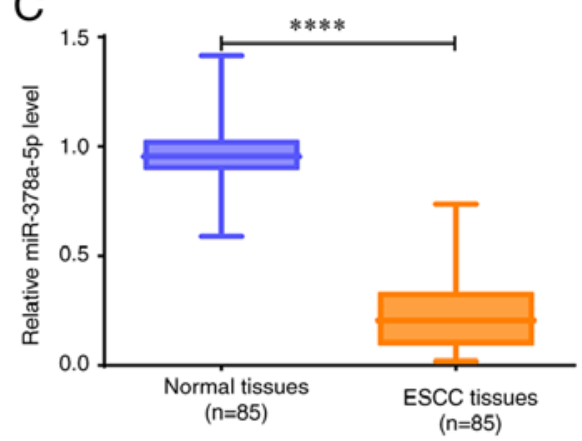

$\mathrm{E}$

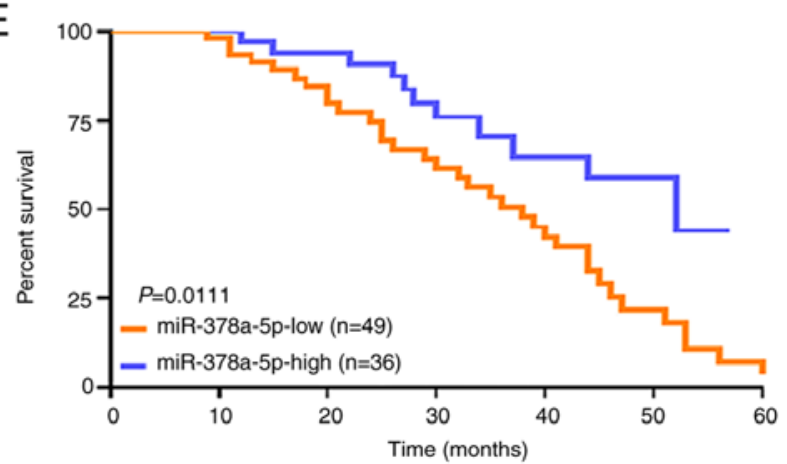

Figure 5. miR-378a-5p expression is low in ESCC tissues. (A) starBase was used to determine the expression of miR-378a-5p in 162 esophageal cancer and 11 normal samples. (B) The GSE43732 Gene Expression Omnibus dataset was used to assess the levels of miR-378a-5p in 119 ESCC and paired normal samples. Data were compared using a paired Student's t-test. (C) RT-qPCR analysis was used to determine the miR-378a-5p levels in 85 ESCC and paired normal samples. Data were analyzed using a Wilcoxon signed rank. (D) RT-qPCR was used to determine the miR-378a-5p levels in various ESCC cell lines (Eca109, KYSE150, KYSE30, KYSE450 and KYSE70) and a normal esophageal epithelial cell line (Het-1A), and the data were compared using a one way ANOVA followed by a Dunnett's post hoc test. (E) A log-rank test was used to analyze the effects of miR-378a-5p expression on the survival rate of patients with $\mathrm{ESCC}$. ${ }^{*} \mathrm{P}<0.05,{ }^{* *} \mathrm{P}<0.01,{ }^{* * *} \mathrm{P}<0.001$, ${ }^{* * * * *} \mathrm{P}<0.0001$. ESCC, esophageal squamous cell carcinoma; miR, microRNA; RT-qPCR, reverse transcription-quantitative PCR.

in osteosarcoma. LINC00514 functioned as a ceRNA by directly absorbing miR-708-5p, and consequently promoting cell proliferation (38). In addition, LINC00514 downregulation suppressed the proliferation, migration and invasion of papillary thyroid cancer cells, which was achieved by sponging miR-204-3p to increase the expression of cell division cycle 23 (19). The present study found that LINC00514 knockdown markedly inhibited the proliferation and invasion of ESCC cells, whereas LINC00514 overexpression promoted the proliferation and invasion of ESCC cells. Further research confirmed that LINC00514 knockdown notably reduced the expression of lipogenesis-related proteins (SPHK1, FASN, ACACA and SCD1) in ESCC cells, and the opposite results were obtained when LINC00514 was upregulated. These data suggest that LINC00514 may participate in the regulation of cell proliferation, invasion and lipogenesis in ESCC cells. However, whether lipogenesis-related proteins participate in the proliferation and invasion of ESCC remains to be determined, and will form the basis of future experiments into the possible biological functions mediated by LINC00514 in ESCC cells.

Based on the close association between the functions of lncRNAs and their subcellular localization (39-41), a subcellular localization assay was performed using RT-qPCR in the present study. It was found that LINC00514 was primarily localized in the cytoplasm of ESCC cells, suggesting that LINC00514 functions as a ceRNA in ESCC. It was also found that LINC00514 directly regulated the miR-378a-5p level in ESCC cells. miRNAs function as negative regulators of downstream target genes by binding to their 3'-untranslated region (42). miR-378a-3p and miR-378a-5p belong to the two mature strands of miR-378a localized at chromosome $5 \mathrm{q} 32$, which was previously known as miR-378 (43). miR-378a has been confirmed to be implicated in the metabolism of lipids and xenobiotics, as well as in lipid storage, the glycolytic pathway and in mitochondrial function $(44,45)$. Pan et al (46) found that miR-378a-5p was reduced in renal tissues and renal cell carcinoma cells, and patients with a high miR-378a-5p level exhibited longer overall survival rates than those of patients with low miR-378a-5p levels. In addition, previous reports have demonstrated that miR-378a-5p is implicated in the regulation of metabolism and angiogenesis $(43,47)$. The present study found low expression of miR-378a-5p in ESCC tissues, and this was closely associated with TNM stage and lymph node metastasis, suggesting that the LINC00514/miR-378a-5p signaling axis may be an important therapeutic target for patients with ESCC.

Lipogenesis contributes to membrane synthesis, provides a source of energy for tumor cells and promotes oncogenic signaling (48); thus, reprogramming lipid metabolism may be a potential therapeutic target in cancer treatment (49). Lipogenesis has been reported to be closely associated with tumor development, progression and metastasis (50-52). SPHK1 is an oncogenic enzyme that phosphorylates 
A

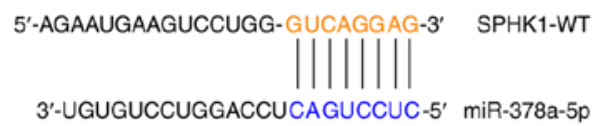

5'-AGAAUGAAGUCCUGG-GUCAGGAG-3' SPHK1-MUT
$\mathrm{B}$

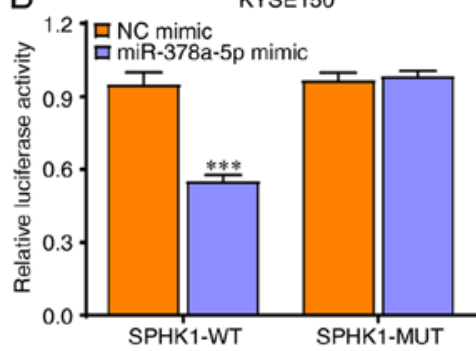

KYSE30

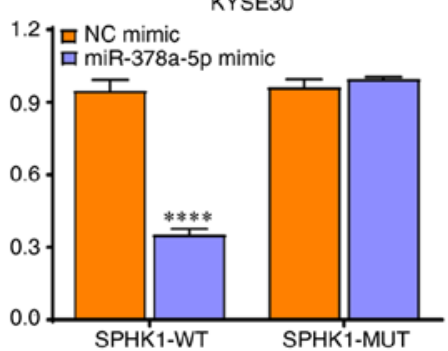

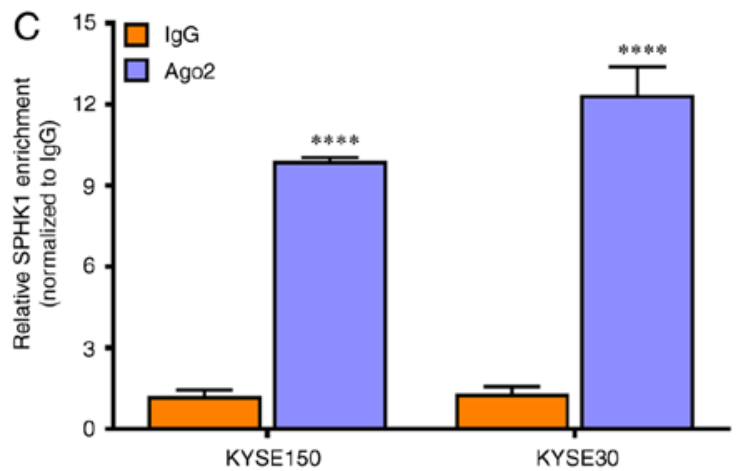
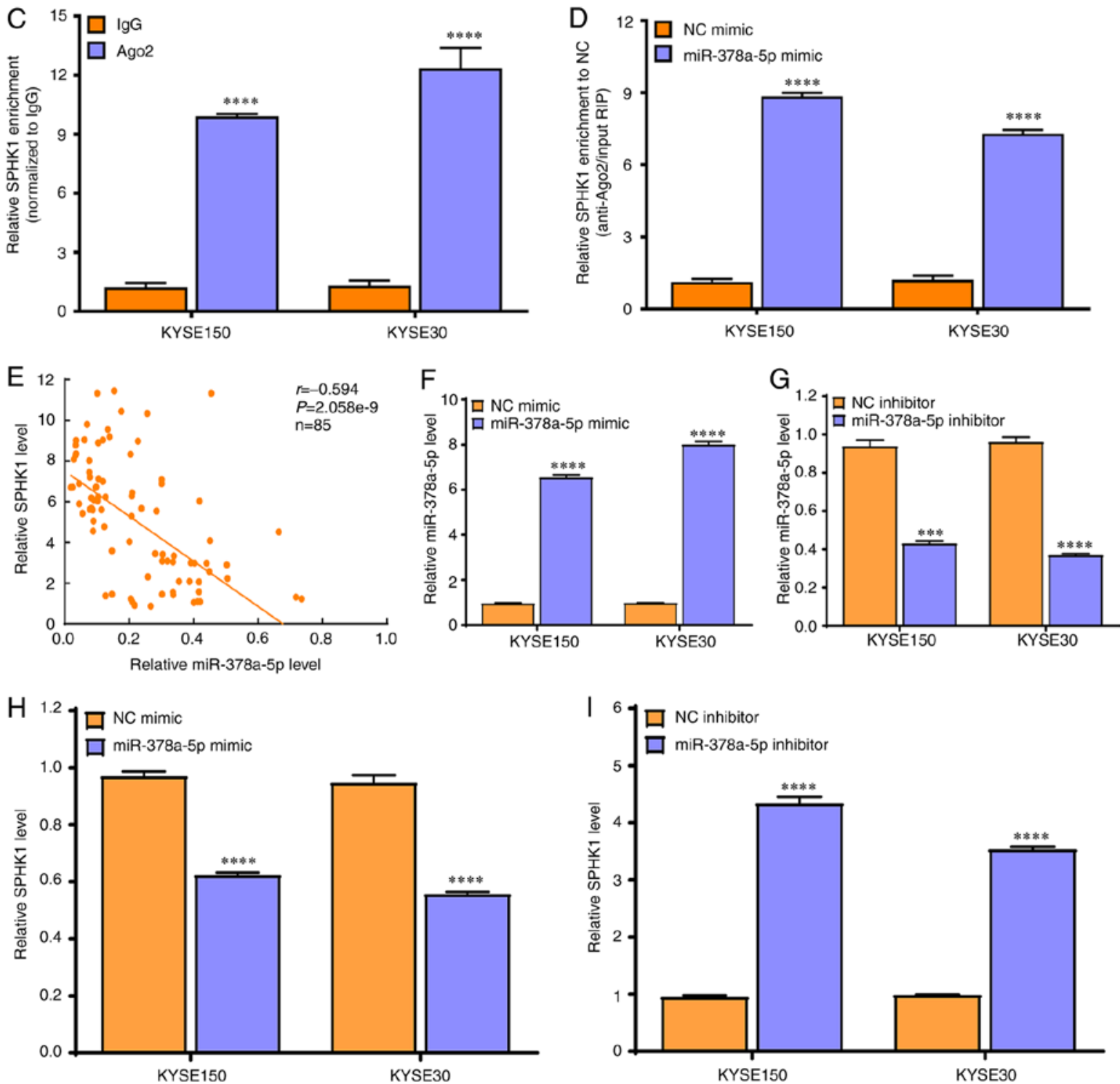

Figure 6. SPHK1 is a direct target of miR-378a-5p in ESCC cells. (A) TargetScan and miRDB both predicted SPHK1 as the downstream target of miR-378a-5p. (B) A dual-luciferase reporter assay was performed to investigate the binding status of SPHK1 and miR-378a-5p in KYSE150 and KYSE30 cells. (C) Ago2-RIP analysis of the enrichment of SPHK1 in RNA-induced silencing complex in KYSE150 and KYSE30 cells. (D) Ago2-RIP analysis of the enrichment of SPHK1 in KYSE150 and KYSE30 cells after transfection with miR-378a-5p mimic. (E) Spearman's rank correlation analysis was used to determine the correlation between miR-378a-5p and SPHK1 expression in 85 ESCC and paired normal samples. (F) miR-378a-5p mimic significantly upregulated the expression of miR-378a-5p in KYSE150 and KYSE30 cells. (G) miR-378a-5p inhibitor markedly downregulated the expression of miR-378a-5p in KYSE150 and KYSE30 cells. (H) miR-378a-5p mimic suppressed the expression of SPHK1 in KYSE150 and KYSE30 cells. (I) miR-378a-5p inhibitor promoted the expression of SPHK1 in KYSE150 and KYSE30 cells. ${ }^{* * *} \mathrm{P}<0.001,{ }^{* * * *} \mathrm{P}<0.0001$. SPHK1, sphingosine kinase 1; ESCC, esophageal squamous cell carcinoma; miR, microRNA; Ago2, argonaute 2; RIP, RNA immunoprecipitation.

sphingosine to produce sphingosine-1-phosphate, and plays a pivotal role in multiple cellular processes $(53,54)$. Increasing evidence has demonstrated that SPHK1 is upregulated in gastric carcinoma (55), colorectal cancer (56) colon cancer (57) and ESCC (58). In the present study, SPHK1 was confirmed as a direct molecular target of miR-378a-5p, and SPHK1 was closely associated with TNM stage, lymph node metastasis and poor prognosis of patients with ESCC. 
A
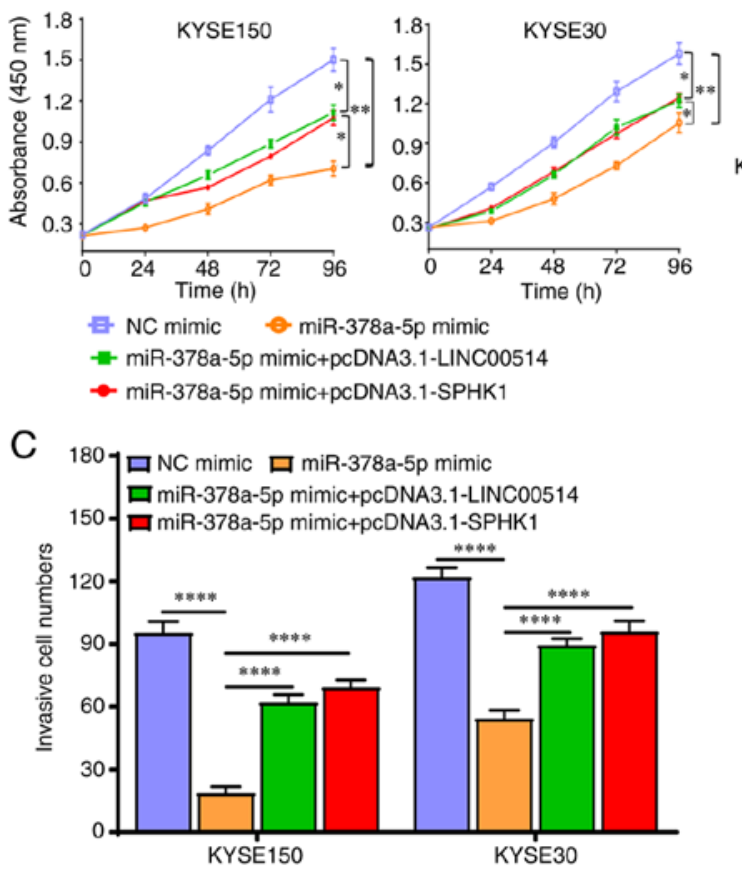

B
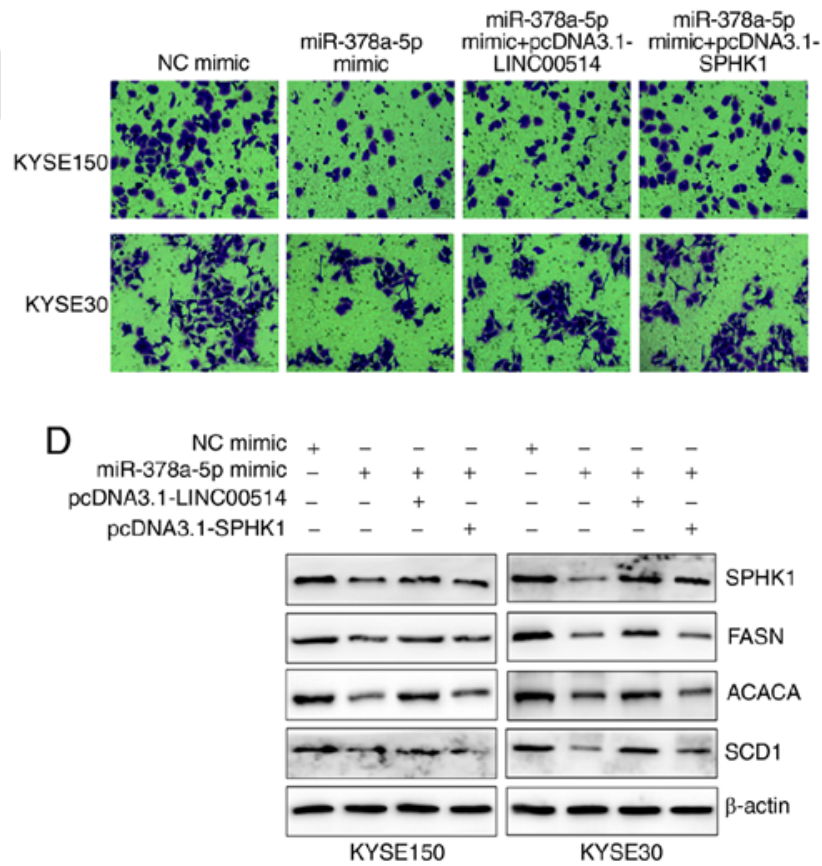

Figure 7. miR-378a-5p exerts biological functions that are dependent on the levels of LINC00514 and SPHK1 in ESCC cells. (A) ESCC cell proliferation was assessed using a CCK-8 assay in the NC mimic, miR-378a-5p mimic, miR-378a-5p mimic + pcDNA3.1-LINC00514 and pcDNA3.1-SPHK1 groups. (B) Cell invasion ability was assessed using a Transwell chamber assay. (C) Quantification of the number of cells that had invaded in each group. Data was compared using a one-way ANOVA followed by a posy-hoc Tukey's post hoc test. (D) Western blotting was used to analyze the expression of SPHK1, FASN, ACACA and SCD1 proteins in various groups. ${ }^{*} \mathrm{P}<0.05,{ }^{* *} \mathrm{P}<0.01,{ }^{* * * * *} \mathrm{P}<0.0001$. SPHK1, sphingosine kinase 1; ESCC, esophageal squamous cell carcinoma; miR, microRNA; LINC00514, long intergenic nonprotein-coding RNA 00514; siRNA, small interfering RNA; NC, negative control; FASN, fatty acid synthetase; ACACA, acetyl-CoA carboxylase $\alpha$; SCD1, stearoyl-CoA desaturase 1.
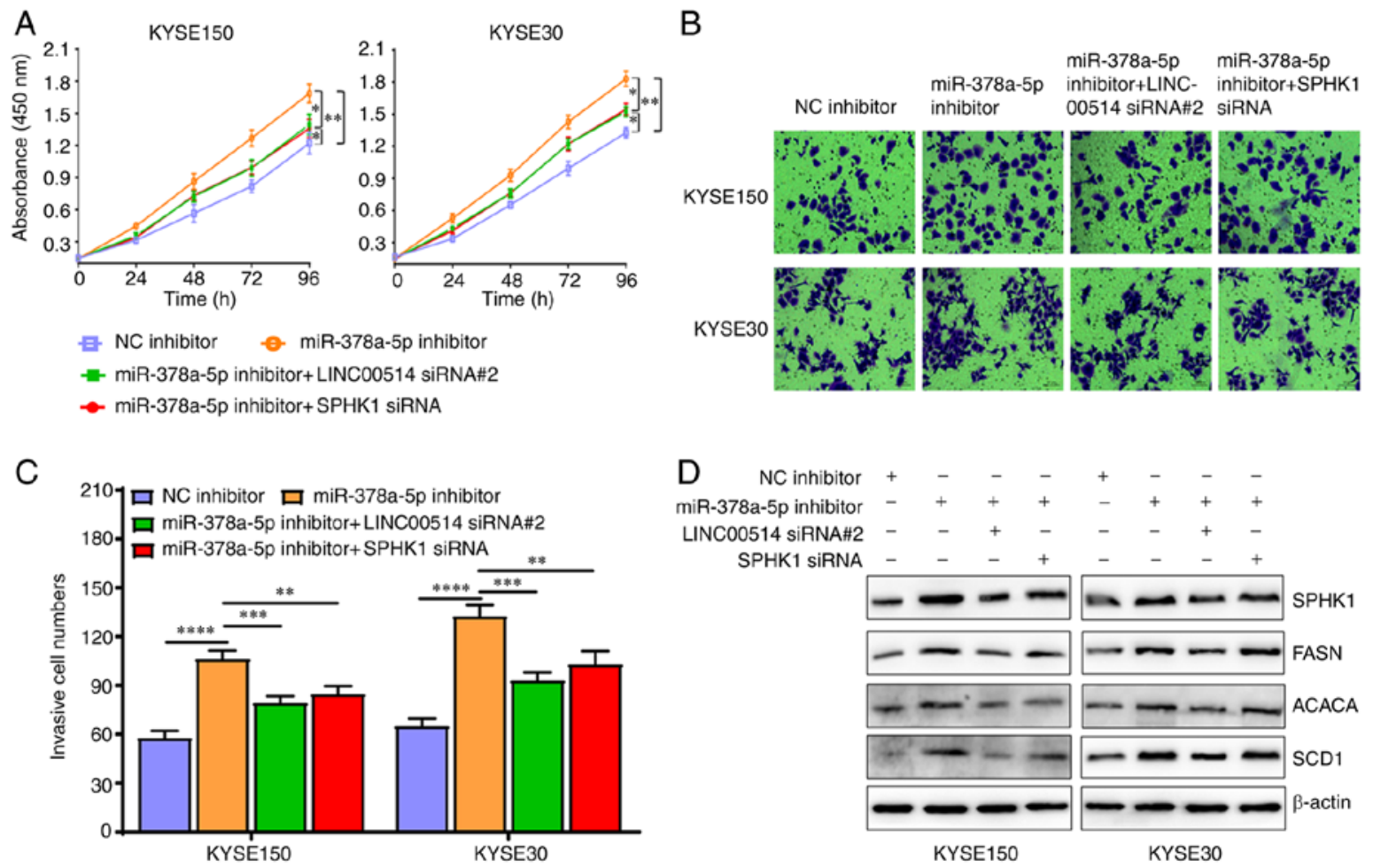

Figure 8. Effects of miR-378a-5p inhiation are partly reversed by the downregulation of LINC00514 and SPHK1 in ESCC cells. (A) ESCC cell proliferation was assessed using a CCK-8 assay in the NC inhibitor, miR-378a-5p inhibitor, miR-378a-5p inhibitor + LINC00514 siRNA2 and SPHK1 siRNA groups. (B) Cell invasive ability was detected using a Transwell chamber assay in the various groups. (C) Quantification of the number of cells that had invaded in the various groups. Data was compared using a one-way ANOVA with a post hoc Tukey's test. (D) Western blot analysis of the expression of SPHK1, FASN, ACACA and SCD1 expression in the various groups. ${ }^{*} \mathrm{P}<0.05,{ }^{* * *} \mathrm{P}<0.01,{ }^{* * *} \mathrm{P}<0.001,{ }^{* * * * *} \mathrm{P}<0.0001$. SPHK1, sphingosine kinase 1; ESCC, esophageal squamous cell carcinoma; miR, microRNA; LINC00514, long intergenic nonprotein-coding RNA 00514; siRNA, small interfering RNA; NC, negative control; FASN, fatty acid synthetase; ACACA, acetyl-CoA carboxylase $\alpha$; SCD1, stearoyl-CoA desaturase 1 . 


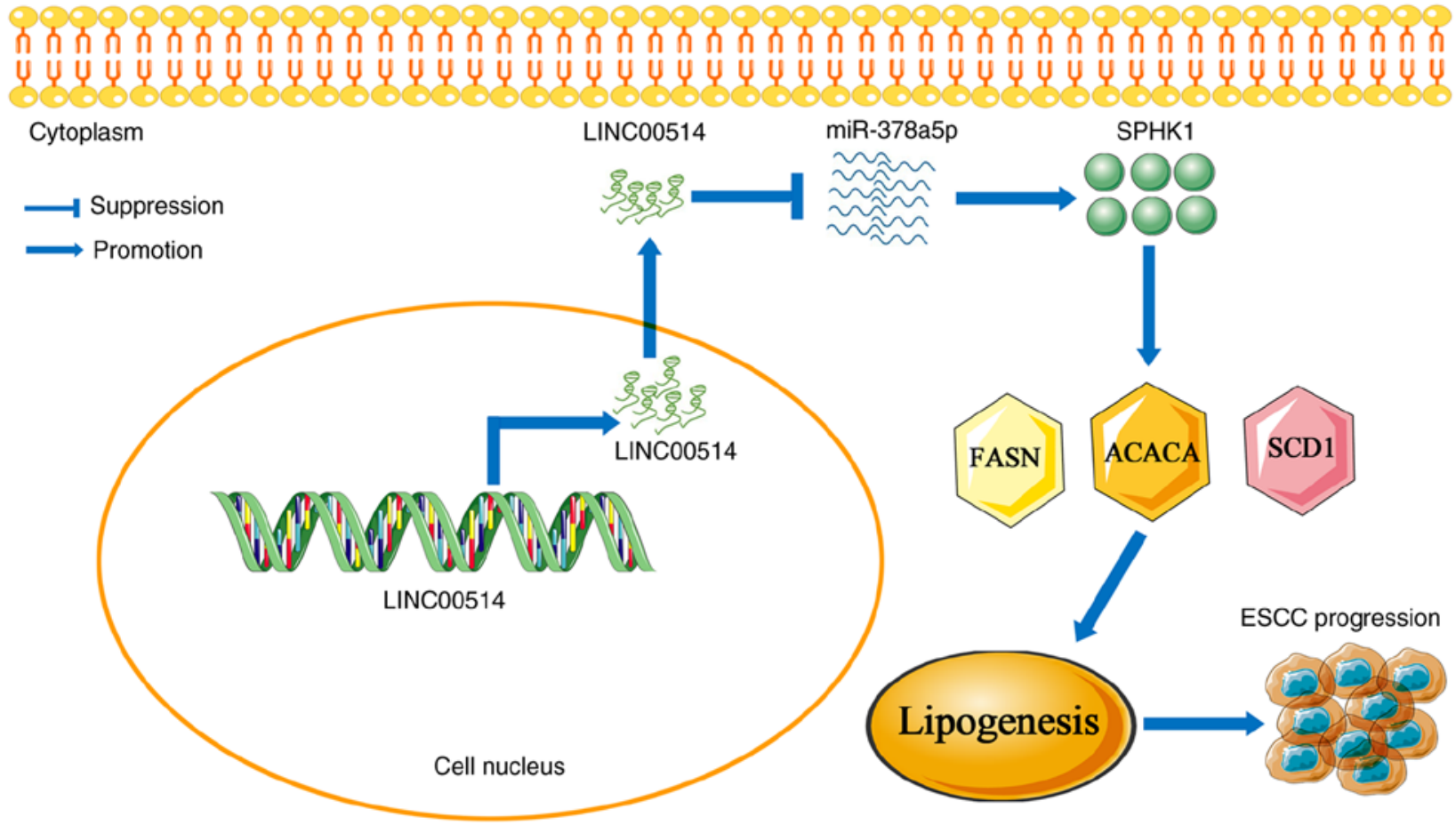

Figure 9. LINC00514 promotes cell proliferation, invasion and lipogenesis by affecting a miR-378a-5p/SPHK1 signaling axis. LINC00514 expression is high in ESCC tissues and cells, and overexpression of LINC00514 reduced the expression of miR-378a-5p in ESCC cells. Further results revealed the upregulation of SPHK1 expression in ESCC cells, coupled with the upregulation of the lipogenesis-related proteins fatty acid synthetase, acetyl-CoA carboxylase $\alpha$ and stearoyl-CoA desaturase, which triggers ESCC lipogenesis and progression. LINC00514, long intergenic nonprotein-coding RNA 00514; ESCC, esophageal squamous cell carcinoma; miR, microRNA; SPHK1, sphingosine kinase 1.

To further clarify the role of the LINC00514/ miR-378a-5p/ SPHK1 signaling axis in ESCC cell proliferation and invasion, CCK-8 and Transwell chamber assays were employed to investigate the function of this axis. The current data revealed that miR-378a-5p mimic significantly suppressed cell proliferation and invasion, and the expression of lipogenesis-related proteins in ESCC cells, which was partly reversed by overexpression of LINC00514 and SPHK1. However, the opposite results were obtained with miR-378a-5p alone or combined with LINC00514 and SPHK1 siRNAs. These results suggest that the LINC00514/miR-378a-5p/SPHK1 signaling axis may be closely associated with ESCC development and progression, and may be a novel and promising therapeutic target for patients with ESCC. Thus, in future studies, the function of the LINC00514/miR-378a-5p/SPHK1 signaling axis will be further assessed in vivo, to lay the foundation for targeting of this signaling axis as a potential therapeutic option in the treamtent of patients with ESCC.

In conclusion, the current data demonstrated that LINC00514 and SPHK1 expression levels were upregulated in ESCC tissues and cells, and this high expression of LINC00514 and SPHK1 was correlated with TNM stage, lymph node metastasis and poor prognosis in patients with ESCC. LINC00514 knockdown inhibited cell proliferation and invasion, and reduced the expression of lipogenesis-related proteins, whereas LINC00514 overexpression accelerated the proliferation and invasion of ESCC cells, and promoted the expression of lipogenesis-related proteins. Mechanistically, LINC00514 functioned as a ceRNA to sponge miR-378a-5p, thereby indirectly upregulating SPHK1 expression, which further promoted the expression of the lipogenesis-related proteins FASN, ACACA and SCD1, and thus promoting ESCC progression. The current data may provide novel evidence for the use of LINC00514/miR-378a-5p/SPHK1 signaling axis-based targeted therapy in patients with ESCC.

\section{Acknowledgements}

We would like to thank Professor Shenglei Li and Associate Professor Yue Xu, Department of Pathology of the First Affiliated Hospital of Zhengzhou University, for their assistance in identification of the ESCC tissues and normal tissues.

\section{Funding}

The present study was supported by the Key R\&D and Promotion Projects in Henan Province (grant no. 182102310380), the Natural Science Foundation of Henan Province (grant no. 182300410377) and the Key Scientific Research Projects of Henan Higher Education Institutions (grant no. 17A180016).

\section{Availability of data and materials}

The datasets used and/or analyzed during the present study are available from the corresponding author on reasonable request.

\section{Authors' contributions}

RTF, XW and HTL conceived and designed the present study. XW, HTL and QZ performed the experiments. XYZ, YQ, GZZ 
and JHD analyzed and interpretated the data. FW and XXY contributed to analysis of the data. RTF wrote the original manuscript. FW and XXY reviewed and revised the manuscript. All authors read and approved the final manuscript. XW and HTL confirm the authenticity of all the raw data.

\section{Ethics approval and consent to participate}

The present study was approved by the Research and Ethics Committee of The First Affiliated Hospital of Zhengzhou University. Informed consent was obtained from all patients.

\section{Patient consent for publication}

Not applicable.

\section{Competing interests}

The authors declare that they have no competing interests.

\section{References}

1. Zhang Y: Epidemiology of esophageal cancer. World J Gastroenterol 19: 5598-5606, 2013.

2. Ferlay J, Soerjomataram I, Dikshit R, Eser S, Mathers C, Rebelo M, Parkin DM, Forman D and Bray F: Cancer incidence and mortality worldwide: Sources, methods and major patterns in GLOBOCAN 2012. Int J Cancer 136: E359-E386, 2015.

3. Stoecklein NH, Hosch SB, Bezler M, Stern F, Hartmann CH, Vay C, Siegmund A, Scheunemann P, Schurr P, Knoefel WT, et al Direct genetic analysis of single disseminated cancer cells for prediction of outcome and therapy selection in esophageal cancer. Cancer Cell 13: 441-453, 2008.

4. Arnold M, Soerjomataram I, Ferlay J and Forman D: Global incidence of oesophageal cancer by histological subtype in 2012 . Gut 64: 381-387, 2015

5. Yang H, Liu H, Chen Y, Zhu C, Fang W, Yu Z, Mao W, Xiang J, Han Y, Chen Z, et al: Neoadjuvant chemoradiotherapy followed by surgery versus surgery alone for locally advanced squamous cell carcinoma of the esophagus (NEOCRTEC5010): A phase III multicenter, randomized, open-label clinical trial. J Clin Oncol 36: 2796-2803, 2018

6. Pickens A and Orringer MB: Geographical distribution and racial disparity in esophageal cancer. Ann Thorac Surg 76 (Suppl): S1367-S1369, 2003.

7. Pennathur A, Gibson MK, Jobe BA and Luketich JD: Oesophageal carcinoma. Lancet 381: 400-412, 2013.

8. Ohashi S, Miyamoto S, Kikuchi O, Goto T, Amanuma Y and Muto M: Recent advances from basic and clinical studies of esophageal squamous cell carcinoma. Gastroenterology 149: $1700-1715,2015$.

9. Morris KV and Mattick JS: The rise of regulatory RNA. Nat Rev Genet 15: 423-437, 2014.

10. Schmitt AM and Chang HY: Long noncoding RNAs in cancer pathways. Cancer Cell 29: 452-463, 2016.

11. Kung JT, Colognori D and Lee JT: Long noncoding RNAs: Past, present, and future. Genetics 193: 651-669, 2013.

12. Ponting CP, Oliver PL and Reik W: Evolution and functions of long noncoding RNAs. Cell 136: 629-641, 2009.

13. Chen MJ, Deng J, Chen C, Hu W, Yuan YC and Xia ZK: lncRNA H19 promotes epithelial mesenchymal transition and metastasis of esophageal cancer via STAT3/EZH2 axis. Int J Biochem Cell Biol 113: 27-36, 2019

14. Liang Y, Chen X, Wu Y, Li J, Zhang S, Wang K, Guan X, Yang K and Bai Y: lncRNA CASC9 promotes esophageal squamous cell carcinoma metastasis through upregulating LAMC2 expression by interacting with the CREB-binding protein. Cell Death Differ 25: 1980-1995, 2018

15. Wu Y, Hu L, Liang Y, Li J, Wang K, Chen X, Meng H, Guan X, Yang $\mathrm{K}$ and Bai Y: Up-regulation of lncRNA CASC9 promotes esophageal squamous cell carcinoma growth by negatively regulating PDCD4 expression through EZH2. Mol Cancer 16: 150, 2017.
16. Li W, Zhang L, Guo B, Deng J, Wu S, LiF, Wang Y,Lu J and Zhou Y: Exosomal FMR1-AS1 facilitates maintaining cancer stem-like cell dynamic equilibrium via TLR7/NFkB/c-Myc signaling in female esophageal carcinoma. Mol Cancer 18: 22, 2019.

17. Tao S, Chen Q, Lin C and Dong H: Linc00514 promotes breast cancer metastasis and M2 polarization of tumor-associated macrophages via Jagged1-mediated notch signaling pathway. J Exp Clin Cancer Res 39: 191, 2020.

18. Yu D, Xu X, Li S and Zhang K: LINC00514 drives osteosarcoma progression through sponging microRNA-708 and consequently increases URGCP expression. Aging 12: 6793-6807, 2020.

19. Li X, Zhong W, Xu Y, Yu B and Liu H: Silencing of lncRNA LINC00514 inhibits the malignant behaviors of papillary thyroid cancer through miR-204-3p/CDC23 axis. Biochem Biophys Res Commun 508: 1145-1148, 2019.

20. Ramnarine VR, Alshalalfa M, MoF,Nabavi N,Erho N, Takhar M, Shukin R, Brahmbhatt S, Gawronski A, Kobelev M, et al: The long noncoding RNA landscape of neuroendocrine prostate cancer and its clinical implications. Gigascience 7: giy050, 2018.

21. Jafari N, Drury J, Morris AJ, Onono FO, Stevens PD, Gao T, Liu J, Wang C, Lee EY, Weiss HL, et al: De novo fatty acid synthesis-driven sphingolipid metabolism promotes metastatic potential of colorectal cancer. Mol Cancer Res 17: 140-152, 2019.

22. Jairajpuri DS, Mohammad T, Adhikari K, Gupta P, Hasan GM, Alajmi MF, Rehman MT, Hussain A and Hassan MI: Identification of sphingosine kinase-1 inhibitors from bioactive natural products targeting cancer therapy. ACS Omega 5: 14720-14729, 2020.

23. Imbert C, Montfort A, Fraisse M, Marcheteau E, Gilhodes J, Martin E, Bertrand F, Marcellin M, Burlet-Schiltz O, Peredo AG, et al: Resistance of melanoma to immune checkpoint inhibitors is overcome by targeting the sphingosine kinase-1. Nat Commun 11: 437, 2020

24. Arends MJ, Fukayama M, Klimstra DS, Lam AKY, Nagtegaal ID, Odze RD, Paradis V, Park YN, Rugge M, Salto-Tellez M, et al: TNM staging of tumours of the oesophagus. WHO classification of tumours of the digestive system. The WHO Classification of Tumours Editorial Board. IARC Press, Lyon, p25, 2019.

25. Paraskevopoulou MD, Vlachos IS, Karagkouni D, Georgakilas G, Kanellos I, Vergoulis T, Zagganas K, Tsanakas P, Floros E, Dalamagas $\mathrm{T}$ and Hatzigeorgiou AG: DIANA-LncBase v2: Indexing microRNA targets on non-coding transcripts. Nucleic Acids Res 44: D231-D238, 2016.

26. Livak KJ and Schmittgen TD: Analysis of relative gene expression data using real-time quantitative PCR and the 2(-Delta Delta $\mathrm{C}(\mathrm{T})$ ) method. Methods 25: 402-408, 2001.

27. Deng SJ, Chen HY, Ye Z, Deng SC, Zhu S, Zeng Z, He C, Liu ML, Huang K, Zhong JX, et al: Hypoxia-induced lncRNA-BX111 promotes metastasis and progression of pancreatic cancer through regulating ZEB1 transcription. Oncogene 37: 5811-5828, 2018

28. Kim J, Piao HL, Kim BJ, Yao F, Han Z, Wang Y, Xiao Z, Siverly AN, Lawhon SE, Ton BN, et al: Long noncoding RNA MALAT1 suppresses breast cancer metastasis. Nat Genet 50: 1705-1715, 2018.

29. Renganathan A and Felley-Bosco E: Long noncoding RNAs in cancer and therapeutic potential. Adv Exp Med Biol 1008: 199-222, 2017.

30. Arun G, Diermeier SD and Spector DL: Therapeutic targeting of long non-coding RNAs in cancer. Trends Mol Med 24: 257-277, 2018 .

31. Li ZX, Zhu QN, Zhang HB, Hu Y, Wang G and Zhu YS: MALAT1: A potential biomarker in cancer. Cancer Manag Res 10: 6757-6768, 2018.

32. Qiu L, Tang Q, Li G and Chen K: Long non-coding RNAs as biomarkers and therapeutic targets: Recent insights into hepatocellular carcinoma. Life Sci 191: 273-282, 2017.

33. Cossu AM, Mosca L, Zappavigna S, Misso G, Bocchetti M, De Micco F, Quagliuolo L, Porcelli M, Caraglia M and Boccellino M: Long non-coding RNAs as important biomarkers in laryngeal cancer and other head and neck tumours. Int J Mol Sci 20: 3444, 2019.

34. Peng Z, Liu C and Wu M: New insights into long noncoding RNÂs and their roles in glioma. Mol Cancer 17: 61, 2018.

35. Chen Y, Bi F, An Y and Yang Q: Identification of pathological grade and prognosis-associated lncRNA for ovarian cancer. J Cell Biochem 120: 14444-14454, 2019.

36. Chao Y and Zhou D: IncRNA-D16366 is a potential biomarker for diagnosis and prognosis of hepatocellular carcinoma. Med Sci Monit 25: 6581-6586, 2019.

37. Yang J, Li C, Mudd A and Gu X: lncRNA PVT1 predicts prognosis and regulates tumor growth in prostate cancer. Biosci Biotechnol Biochem 81: 2301-2306, 2017. 
38. Mi LD, Sun CX, He SW and Du GY: SP1-Induced upregulation of lncRNA LINC00514 promotes tumor proliferation and metastasis in osteosarcoma by regulating miR-708. Cancer Manag Res 12: 3311-3322, 2020

39. Chaumeil J, Le Baccon P, Wutz A and Heard E: A novel role for Xist RNA in the formation of a repressive nuclear compartment into which genes are recruited when silenced. Genes Dev 20 2223-2237, 2006.

40. Rinn JL, Kertesz M, Wang JK, Squazzo SL, Xu X, Brugmann SA, Goodnough LH, Helms JA, Farnham PJ, Segal E and Chang HY: Functional demarcation of active and silent chromatin domains in human HOX loci by noncoding RNAs. Cell 129: 1311-1323, 2007.

41. Cesana M, Cacchiarelli D, Legnini I, Santini T, Sthandier O, Chinappi M, Tramontano A and Bozzoni I: A long noncoding RNA controls muscle differentiation by functioning as a competing endogenous RNA. Cell 147: 358-369, 2011.

42. Bartel DP: MicroRNAs: Genomics, biogenesis, mechanism, and function. Cell 116: 281-297, 2004.

43. Krist B, Florczyk U, Pietraszek-Gremplewicz K, Jozkowicz A and Dulak J: The role of miR-378a in metabolism, angiogenesis, and muscle biology. Int J Endocrinol 2015: 281756, 2015.

44. Carrer M, Liu N, Grueter CE, Williams AH, Frisard MI, Hulver MW, Bassel-Duby R and Olson EN: Control of mitochondrial metabolism and systemic energy homeostasis by microRNAs 378 and 378*. Proc Natl Acad Sci USA 109: $15330-15335,2012$.

45. Eichner LJ, Perry MC, Dufour CR, Bertos N, Park M, St-Pierre J and Giguère V: miR-378(*) mediates metabolic shift in breast cancer cells via the PGC-1beta/ERRgamma transcriptional pathway. Cell Metab 12: 352-361, 2010.

46. Pan X, Zhao L, Quan J, Liu K, Lai Y, Li Z, Zhang Z, Xu J, Xu W, Guan X, et al: miR-378a-5p acts as a tumor suppressor in renal cell carcinoma and is associated with the good prognosis of patients. Am J Transl Res 11: 2207-2218, 2019.

47. Cui Z, Liu QL, Sun SQ, Jiao K, Liu DR, Zhou XC and Huang L: miR-378a-5p inhibits angiogenesis of oral squamous cell carcinoma by targeting KLK4. Neoplasma 67: 85-92, 2020.

48. Zadra G, Photopoulos C and Loda M: The fat side of prostate cancer. Biochim Biophys Acta 1831: 1518-1532, 2013.

49. Cheng C, Geng F, Cheng X and Guo D: Lipid metabolism reprogramming and its potential targets in cancer. Cancer Commun (Lond) 38: 27, 2018
50. Ishay-Ronen D, Diepenbruck M, Kalathur RKR, Sugiyama N, Tiede S, Ivanek R, Bantug G, Morini MF, Wang J, Hess C and Christofori G: Gain Fat-lose metastasis: Converting invasive breast cancer cells into adipocytes inhibits cancer metastasis. Cancer Cell 35: 17-32.e6, 2019.

51. Porporato PE, Payen VL, Baselet B and Sonveaux P: Metabolic changes associated with tumor metastasis, part 2: Mitochondria, lipid and amino acid metabolism. Cell Mol Life Sci 73: 1349-1363, 2016.

52. Tousignant KD, Rockstroh A, Taherian Fard A, Lehman ML, Wang C, McPherson SJ, Philp LK, Bartonicek N, Dinger ME, Nelson CC and Sadowski MC:Lipid uptake is an androgen-enhanced lipid supply pathway associated with prostate cancer disease progression and bone metastasis. Mol Cancer Res 17: 1166-1179, 2019.

53. Li W, Yu CP, Xia JT, Zhang L, Weng GX, Zheng HQ, Kong QL, $\mathrm{Hu}$ LJ, Zeng MS, Zeng YX, et al: Sphingosine kinase 1 is associated with gastric cancer progression and poor survival of patients. Clin Cancer Res 15: 1393-1399, 2009.

54. Facchinetti MM, Gandini NA, Fermento ME, Sterin-Speziale NB, Ji Y, Patel V, Gutkind JS, Rivadulla MG and Curino AC: The expression of sphingosine kinase-1 in head and neck carcinoma. Cells Tissues Organs 192: 314-324, 2010.

55. Wang Z, Qu H, Gong W and Liu A: Up-regulation and tumor-promoting role of SPHK1 were attenuated by miR-330-3p in gastric cancer. IUBMB Life 70: 1164-1176, 2018.

56. Bae GE, Do SI, Kim K, Park JH, Cho S and Kim HS: Increased sphingosine kinase 1 expression predicts distant metastasis and poor outcome in patients with colorectal cancer. Anticancer Res 39: 663-670, 2019.

57. Liu SQ, Su YJ, Qin MB, Mao YB, Huang JA and Tang GD: Sphingosine kinase 1 promotes tumor progression and confers malignancy phenotypes of colon cancer by regulating the focal adhesion kinase pathway and adhesion molecules. Int J Oncol 42: 617-626, 2013.

58. Nemoto M, Ichikawa H, Nagahashi M, Hanyu T, Ishikawa T, Kano Y, Muneoka Y and Wakai T: Phospho-Sphingosine Kinase 1 expression in lymphatic spread of esophageal squamous cell carcinoma. J Surg Res 234: 123-131, 2019.

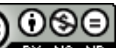

This work is licensed under a Creative Commons Attribution-NonCommercial-NoDerivatives 4.0 International (CC BY-NC-ND 4.0) License. 